\title{
Nowcasting de Inversión. Una estimación en tiempo real con indicadores de alta frecuencia
}

\author{
Fiorella Dogliolo
}

\author{
Tesis de Maestría \\ Maestría en Economía \\ Universidad Nacional de La Plata
}

Director de Tesis: María Lorena Garegnani

Fecha de defensa: 23 de marzo de 2018

Códigos JEL: C22, C53, E37 


\title{
$\underline{\text { Resumen }}$
}

En el presente trabajo se realizó una estimación en tiempo real de la evolución de la Inversión a partir de un conjunto amplio de indicadores económicos de alta frecuencia, lo que se conoce en la literatura como Nowcasting. Para realizar el Nowcast se consideraron tres grupos de indicadores de frecuencia mensual y mediante modelos de factores dinámicos se pronosticó el crecimiento trimestral de la Inversión. Adicionalmente, se realizó un ejercicio de pooling o combinación de pronósticos. A partir del test de Giacomini y White se pudo concluir que los modelos de factores y sus combinaciones exhiben una mejor capacidad predictiva en relación a un modelo AR(1) considerado como benchmark, y que la inclusión de un mayor número de indicadores no necesariamente mejora la performance del pronóstico.

\begin{abstract}
$\underline{\text { Abstract }}$
In this paper I present a real-time estimation of the evolution of the Investment, constructed from a broad set of high frequency economic indicators: known in the literature as Nowcasting. The Nowcast exercise was developed considering three groups of monthly indicators throughout dynamic factor models to forecast Investment growth. Additionally, I conducted a forecast-pooling exercise. Using the Giacomini and White test it was possible to conclude that factor models and the pooling exhibit a better relative predictive capacity than an AR (1) model considered as a benchmark. Furthermore, the inclusion of more indicators does not necessarily improve the predictive capacity.
\end{abstract}

Palabras clave: Nowcasting, modelos de factores dinámicos, pronósticos real-time, pooling de pronósticos 


\section{Contenido}

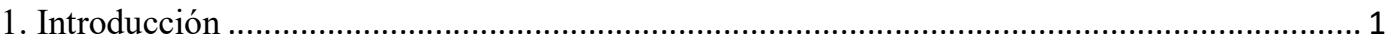

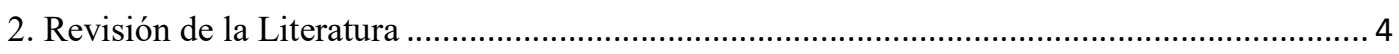

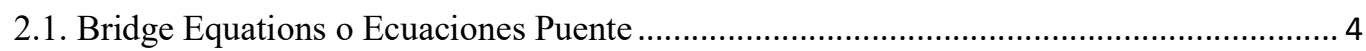

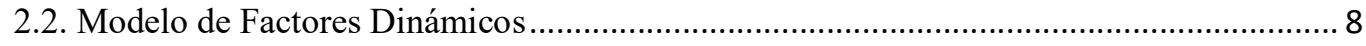

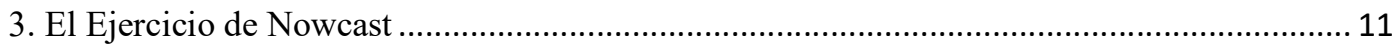

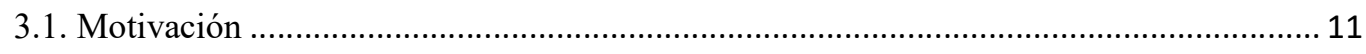

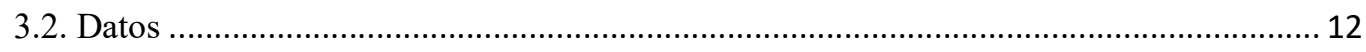

3.3. Metodología de Estimación: Modelo de Factores Dinámicos ........................................... 16

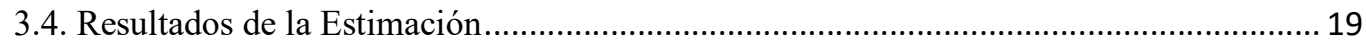

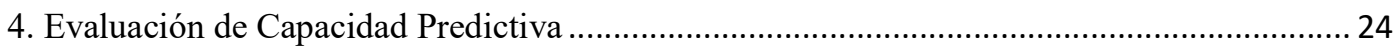

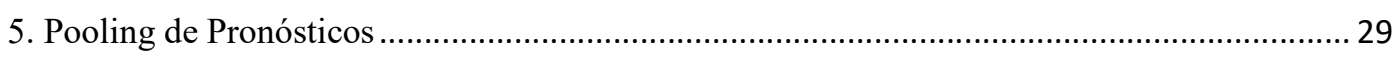

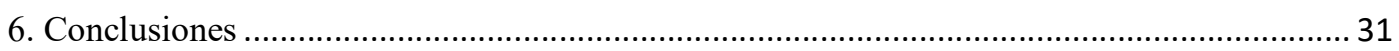

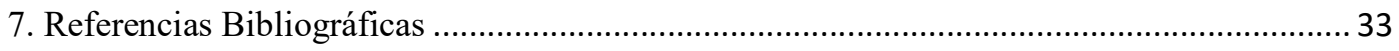

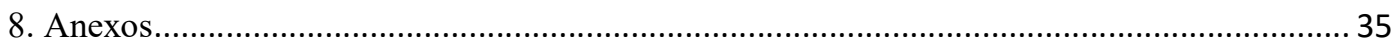

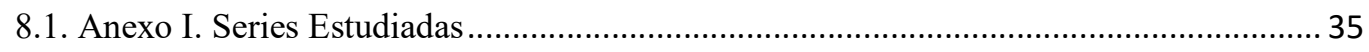

8.2. Anexo II. Coeficiente de Correlación......................................................................... 38

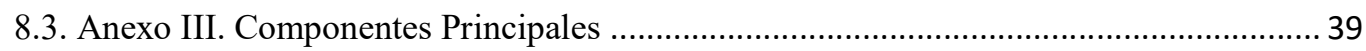

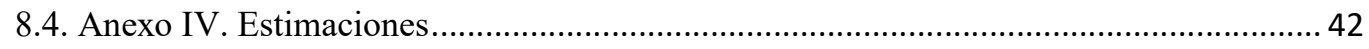




\section{$\underline{\text { 1. Introducción }}$}

Conocer la dinámica de la Inversión, una de las principales variables económicas, es fundamental para la toma de decisiones de los agentes económicos y de los responsables de la política económica.

Sin embargo, el conocimiento del desempeño de la Inversión - tal como ocurre con el resto de los componentes de la demanda agregada publicados en Cuentas Nacionales por INDEC - ocurre con rezago y no es lo suficientemente oportuno para la toma de decisiones, ya que la Inversión es una variable que se difunde con frecuencia trimestral y exhibe un retraso en su publicación cercano a los tres meses de finalizado el trimestre. Esta situación puede ser de particular relevancia cuando se está frente a un cambio de tendencia y la falta de información puede generar un retraso en la implementación de políticas contracíclicas o de incentivo a la Inversión.

Por otro lado, existe una gran cantidad de indicadores económicos de alta frecuencia con frecuencia mensual, quincenal, semanal, o incluso diaria- que pueden ser de gran utilidad para dar cuenta del comportamiento actual de la Inversión y de un posible cambio en la tendencia de la misma. No obstante, producto justamente de la frecuencia, estos indicadores pueden contener ruido que afecta a la información y a la posibilidad de distinguir las señales que brinda la misma. En este sentido, si bien contar con un mayor número de indicadores podría favorecer a tener mayor y mejor información, puede ser que las señales que manifiestan sean heterogéneas y contradictorias, haciendo necesaria alguna metodología de agregación estadística que brinde una única respuesta.

Dada la falta de datos a tiempo, el objetivo del presente trabajo es realizar una estimación en tiempo real de la evolución de la Inversión a partir de un conjunto amplio de indicadores económicos. En particular, se pretende estimar el crecimiento trimestral de la Inversión durante el transcurso del mismo trimestre mediante la utilización de información de alta frecuencia, lo que se conoce en la literatura como Nowcasting. El Nowcasting - definido como la predicción del presente, el futuro muy cercano y el pasado muy reciente (Giannone et al., 2008; Banbura et al., 2013) — ha demostrado ser una herramienta útil para superar el problema de contar con información válida a distintas frecuencias. Este enfoque genera una estimación en tiempo real, dado que se actualiza continuamente a medida que se difunde la nueva información utilizada a tal fin. 
La metodología de Nowcasting — término que surge de las palabras now (ahora) y forecasting (pronóstico) - se origina en la meteorología y su aplicación en la economía se ha expandido fuertemente en los últimos diez años. La literatura ha avanzado en el desarrollo de diversos modelos econométricos para trabajar con estimaciones en las que intervienen variables a diversas frecuencias. Entre las técnicas más utilizadas se encuentran las ecuaciones puentes o bridge equations y los modelos de factores dinámicos, siendo también empleados los modelos de MIxed DAta Sampling (MIDAS), modelos VARs, entre otros.

La aplicación de esta metodología se concentra en obtener estimaciones del Producto Interno Bruto (PIB), principalmente entre los bancos centrales. Tal es el caso del Banco de Inglaterra, la Reserva Federal (Nueva York, Atlanta, Chicago, St. Louis), el Banco Central Europeo, el Banco Central de Irlanda, el Banco de Noruega, el Banco de Francia, el Banco de España, el Banco Central de Turquía, el Banco Central de Sudáfrica, entre otros. El Banco Central de Argentina también utiliza esta metodología para tener una predicción anticipada del crecimiento del PIB (Blanco, D'Amato, Dogliolo y Garegnani, 2017).

El Nowcasting, utilizado fundamentalmente para realizar predicciones del PIB, también puede ser aplicado para realizar estimaciones de otras variables de baja frecuencia publicadas con un sustancial rezago, como la Inversión, haciendo uso de información oportuna de alta frecuencia (según lo sugerido por Banbura et al., 2010). Sin embargo, no hay amplia difusión de trabajos realizados al respecto. Se conoce un documento de trabajo del Banco de Indonesia (Tardsidin, Idham y Robbi Nur Rakhman, 2017), en que utilizan el Nowcasting para realizar una estimación de la Inversión mediante un modelo de factores dinámicos. Por otro lado, si bien algunos bancos centrales también realizan estimaciones de la Inversión, lo hacen como parte del proceso de estimación de los componentes de la demanda agregada que utilizan para el Nowcasting del PIB. En este sentido, la Reserva Federal de Atlanta (Higgins, 2014) y el Banco de Israel (Krief, 2011), entre otros, estiman la Inversión mediante bridge equations a partir de indicadores económicos mensuales, y la utilizan como insumo de sus predicciones del Producto.

De este modo, el presente trabajo representa un aporte a la literatura existente hasta el momento. Particularmente en el caso de Argentina, en que no se cuenta con ningún indicador parcial adelantado de la Inversión, la estimación que surge de este trabajo permitiría obtener información temprana sobre el desempeño de esta variable. Así, los 
hacedores de política podrían tener un conocimiento en tiempo real de la Inversión, lo que es fundamental para la implementación oportuna de políticas de incentivo a la Inversión y de políticas contracíclicas. En este sentido, el Nowcast de Inversión es una herramienta que permitiría conocer el desempeño de la Inversión y favorecería a una asignación eficiente de los recursos que permita lograr un crecimiento de la economía sostenible a largo plazo.

Asimismo, contar con una estimación de la Inversión es de gran utilidad para los modelos de equilibrios general, que hacen uso de modelos satélites de las principales variables macroeconómicas y son ampliamente utilizados en los bancos centrales y en el sistema de la Reserva Federal para la toma de decisiones en la implementación de la política monetaria.

El trabajo consistió, en una primera instancia, en realizar una selección y recopilación de los indicadores económicos a utilizar. Luego del estudio de las series y de realizarles las transformaciones necesarias (desestacionalización, diferenciación o eliminación de tendencia para trabajar con series estacionarias), se utilizó como criterio de elección de las series a incluir en la estimación la existencia de una correlación contemporánea significativa entre la tasa de crecimiento de cada una de las variables y la tasa de crecimiento de la Inversión. Posteriormente, se consideraron diversos conjuntos de indicadores - de acuerdo con el valor del coeficiente de correlación — para luego estimar los factores comunes a cada uno de los grupos y utilizarlos, mediante diversos modelos de factores dinámicos, para predecir el crecimiento de la Inversión. Finalmente, mediante el test de Giacomini y White (Giacomini y White, 2003), se analizó la capacidad predictiva relativa de los modelos de factores y se los comparó a su vez con un benchmark (modelo autoregresivo - AR—), y un pooling de pronósticos.

El documento se organiza de la siguiente manera. En la sección II se realiza una breve descripción de la literatura. En la Sección III.1 se introduce el ejercicio de Nowcasting realizado y su motivación. Los datos recolectados, las transformaciones aplicadas a las series y el criterio de selección de los indicadores económicos a incluir en la estimación de la Inversión se presentan en la Sección III.2. La metodología de estimación utilizada se explica en la Sección III.3, mientras que en la Sección III.4 se muestran los resultados de pronóstico obtenidos con los diversos modelos. En la Sección IV se analiza la capacidad predictiva de los modelos y en la sección V se presenta un ejercicio de pooling de pronósticos. Finalmente, en la sección VI se realizan las conclusiones. 


\section{$\underline{\text { 2. Revisión de la Literatura }}$}

Los agentes económicos $\mathrm{y}$, principalmente, los hacedores de política necesitan tener un conocimiento sobre el estado actual de la economía en tiempo real y sobre su desarrollo próximo esperado. Sin embargo, sólo disponen de información incompleta a la hora de la toma de decisiones. Por ejemplo, uno de los principales indicadores macroeconómicos de actividad - el PIB - se comunica de manera trimestral y se publica con un considerable rezago (diez semanas después de finalizado el trimestre), al tiempo que se encuentran disponibles una gran cantidad de indicadores adelantados o coincidentes con el ciclo económico de frecuencia mensual o aún mayor (quincenal, semanal o diaria).

Esta situación se plantea como un desafío para los pronosticadores al realizar la estimación, dado que se cuenta con información incompleta y desbalanceada. El desbalanceo en la base de datos puede provenir de tener información de distinta frecuencia y del hecho que los retrasos en la publicación de los diversos indicadores generan información faltante para algunas variables al final de la muestra (denominado esto último "ragged -edge" o borde irregular).

En este escenario, la literatura de pronóstico ha avanzado en los últimos años en el desarrollo de una vasta cantidad de modelos econométricos para trabajar con estimaciones en las que intervienen un gran conjunto de datos y variables de diversas frecuencias y fechas de publicación.

\subsection{Bridge Equations o Ecuaciones Puente}

Uno de los primeros enfoques econométricos para trabajar con datos de frecuencias mixtas fue el uso de bridge equations o ecuaciones puente. Este método es ampliamente empleado en varios bancos centrales y en diversas instituciones, especialmente para el Nowcasting y el pronóstico de corto plazo.

La técnica de bridge models permite computar estimaciones tempranas de la variable de baja frecuencia utilizando indicadores de alta frecuencia. Bridge equations son regresiones lineales que unen ("bridge o puente") las variables de alta frecuencia, como 
la producción industrial, las ventas minoristas o las importaciones de bienes de capital, entre otras, con las variables de baja frecuencia, como el crecimiento trimestral del PIB o la Inversión en nuestro caso, proveyendo alguna estimación del desempeño actual o de corto plazo de esta variable con anterioridad a su publicación. Los modelos bridge requieren que los indicadores incluidos contengan información actualizada, esto es que el conjunto de todos los regresores sea conocido durante el período de proyección.

Varias especificaciones se incluyen dentro de esta metodología, por lo que puede haber diferencias de acuerdo con cada trabajo, pero en términos generales la aplicación de este enfoque consta de sucesivas etapas. Tomando por caso la proyección del PIB, dado que los indicadores son mensuales y generalmente están parcialmente disponibles durante el período de estimación, la proyección del crecimiento trimestral del PIB es obtenida en varios pasos. Primero, puede ser necesario proyectar los indicadores mensuales para lo que resta del trimestre, que puede ser realizado mediante un modelo de serie de tiempo univariado, y luego se los agrega para obtener el correspondiente valor trimestral de modo que los indicadores mensuales tengan la misma frecuencia que la variable de baja frecuencia. En esta instancia, puramente empírica, es necesario tener conocimiento de cómo es generada y expresada la información contenida en la variable y también es relevante el tipo de variable de que se trate. En el caso de los flujos, la agregación generalmente se realiza como promedio o suma de las variables de frecuencia mensual, mientras que en el caso de las variables stock muchas veces se promedia o se considera el último valor de la variable.

En segundo lugar, los valores agregados son utilizados como regresores de la bridge equation, la que permite obtener los pronósticos del crecimiento del PIB (en nuestro caso la Inversión). Generalmente, se estiman modelos bivariados autoregresivos de rezagos distribuidos para cada uno de los indicadores. De este modo, el modelo bridge a ser estimado para $x_{j}$ consiste en:

$$
y_{t}^{Q}=\alpha+\sum_{i=1}^{p} \alpha_{i} y_{t-i}^{Q}+\sum_{i=0}^{p} \beta_{i} x_{j t-i}^{Q}+\mu_{t}
$$

Donde $y_{t}^{Q}$ es el crecimiento trimestral del PIB, $\alpha$ es una constante, $x_{j}^{Q}$ corresponde al jésimo indicador mensual agregado en una frecuencia trimestral para ser homogéneo con el PIB, $\mu_{t}$ es el término de error que tiene una distribución normal y $p$ denota el número 
de rezagos en la bridge equation. El máximo rezago $(p)$ se determina de acuerdo con la metodología de general a particular o con algún criterio de información, siendo el máximo número de rezagos igual a cuatro en el caso de las variables trimestrales.

Luego, los pronósticos generados por los $n$ indicadores individuales pueden ser agregados considerando distintos criterios de ponderación para obtener un único pronóstico del PIB para el período corriente. Básicamente, el pooling o agregación de pronósticos se implementa con la siguiente fórmula:

$$
\widehat{y_{t}}=\sum_{i=1}^{n} w_{i} f_{i, t} \quad \operatorname{con} \sum_{i=1}^{n} w_{i}=1
$$

Siendo $\widehat{y_{t}}$ la combinación de los pronósticos, $w_{i}$ el ponderador o peso asignado a los pronósticos individuales basados en el indicador $\mathrm{i}, f_{i, t}$.

Como se describe en Drechsel y Maurin (2008), se han desarrollado diversos esquemas de ponderadores, $w_{i}$, además de utilizar la misma ponderación para todos los pronósticos.

Pueden ponderarse de acuerdo con el poder explicativo relativo dentro de la muestra $\left(R^{2}\right)$, combinando de este modo los pronósticos de acuerdo con el ajuste de cada modelo.

$$
w_{i}=\frac{R_{i}^{2}}{\sum_{j=1}^{n} R_{j}^{2}}
$$

También pueden ponderarse los pronósticos de acuerdo con el desempeño fuera de la muestra, ponderándose de acuerdo con la inversa del error de pronóstico individual (la raíz cuadrada del error cuadrático medio-RMSE por su sigla en inglés-).

$$
w_{i t}=\frac{m_{i t}^{-1}}{\sum_{j=1}^{n} m_{j t}^{-1}} ; \quad \text { donde } m_{i t}=\sqrt{\frac{\sum_{t=T+1}^{T+h}\left(\widehat{y_{l t}}-y_{t}\right)^{2}}{h}}
$$

Otros mecanismos alternativos de ponderación pueden ser aquellos que surgen de métodos Bayesianos.

Los modelos de bridge equations han sido de los primeros métodos utilizados en la metodología de Nowcasting para conocer el estado actual de la economía, haciendo uso de los indicadores mensuales disponibles. Estos estudios se han realizado en diferentes economías. Un resultado común a estos trabajos es que la explotación de información dentro del período de estimación reduce los errores de pronóstico en la mayoría de los 
casos. La aplicación abarca tanto a modelos por el lado de la oferta como por el lado de la demanda.

Por ejemplo, Baffigi, Golinelli y Pargi (2004) examinan la habilidad de pronóstico de los bridge models para pronosticar el crecimiento del PIB en la zona del euro. En el trabajo, los modelos bridge son estimados para el PIB y sus componentes, tanto para la euro-area en su conjunto como para las principales economías de la zona. La performance de corto plazo (uno y dos trimestres hacia adelante) es comparada contra los modelos benchmark univariados y multivariados, y también es contrastada con pequeños modelos estructurales. Los resultados son contundentes, al señalar que los modelos bridge siempre superan a los benchmark, cuando al menos alguno de los indicadores esté disponible durante el horizonte de pronóstico. Además, encuentran que el PIB de la zona del euro y sus componentes se predicen con mayor precisión agregando pronósticos nacionales.

Diron (2008) hace uso de los modelos bridge con datos de la zona del euro para realizar una evaluación de los errores de pronóstico ante la revisión de datos. En particular, en un ejercicio con información de cuatro años, el documento proporciona estimaciones de los errores de pronóstico para el crecimiento del PIB real de la zona del euro en condiciones de tiempo real, y evalúa el impacto de las revisiones de datos sobre las predicciones a corto plazo del crecimiento real del PIB. Encuentra que las proyecciones tienden a ser similares, ya sea que se basen en datos preliminares o revisados, y que por consiguiente los RMSE basados en ejercicios en tiempo real y pseudo tiempo real son bastante semejantes, y en ambos casos más pequeños que los que surgen de los pronósticos de un modelo AR del PBI, considerado como benchmark. Es decir, encuentran que los pronósticos de corto plazo basados en bridge equations son informativos.

Un estudio de Barhoumi, Darné, Ferrara y Pluyaud (2011) presenta un modelo para predecir la tasa de crecimiento trimestral del Producto de Francia. Los autores emplean bridge equations para pronosticar cada componente del PIB, por el lado de la oferta y por el lado de la demanda, y seleccionan las variables explicativas de frecuencia mensual dentro de un gran conjunto de indicadores. Llevan a cabo un ejercicio de pronóstico mediante ventanas móviles para evaluar la performance en la predicción del PIB, tomando en cuenta los retrasos en la publicación de los datos para generar pronósticos en pseudoreal time. Encuentran que estos modelos resultan ser superiores al benchmark en términos de pronóstico. 
Bencivelli, Marcellino y Moretti (2012) investigan la performance de los modelos bridge en un contexto donde la selección de las variables se realiza mediante un modelo Bayesiano. En el trabajo seleccionan en cada período el mejor conjunto de predictores de acuerdo con la función de distribución de probabilidades posterior del espacio del modelo. Esto permite hacer uso de un conjunto más amplio de indicadores que los que habitualmente se utilizan en los bridge models tradicionales. En un ejercicio pseudo realtime para la zona del euro y para Alemania, Francia e Italia, encuentran que los modelos construidos usando los promedios Bayesianos superan en capacidad predictiva a los bridge models tradicionales, basados en una selección fija y reducida de variables.

\subsection{Modelo de Factores Dinámicos}

Los modelos de factores dinámicos (dinamic factor models) resumen la información contenida en un conjunto amplio de datos utilizando un número reducido de factores, cuyo comportamiento dinámico está especificado como un proceso autoregresivo.

Los modelos de factores han sido empleados de manera generalizada en la literatura para trabajar con datos de distintas frecuencias, y se ha demostrado que permiten explotar una gran cantidad de información y obtener pronósticos más precisos que mediante otras metodologías.

Uno de los modelos más utilizados en la literatura de Nowcasting y en el que se basan los desarrollos de los modelos de factores es el de Stock y Watson (2002). Los autores emplearon estos modelos para extraer el estado no observado de la economía de Estados Unidos, con indicadores mensuales del ciclo combinados con el PIB real trimestral.

Particularmente, su metodología consiste en un modelo en que $y_{t+1}$ es la variable que se quiere pronosticar, y $X_{t}$ es el vector $\mathrm{N}$-dimensional de variables observables utilizadas como predictores. Se supone que $X_{t}, y_{t+1}$ admiten una representación a través de un modelo de factores dinámicos, con $r$ factores dinámicos comunes no observables $f_{t}$, de la forma:

$$
\begin{gathered}
y_{t+1}=\beta(L) f_{t}+\gamma(L) y_{t}+\varepsilon_{t+1} \\
X_{i t}=\lambda_{i}(L) f_{t}+e_{i t}
\end{gathered}
$$


para $i=1, \ldots, N$, donde $e_{t}=\left(e_{1 t}, e_{2 t}, \ldots, e_{N t}\right)^{\prime}$ es un vector $N \times 1$ de las perturbaciones idiosincráticas, y $\lambda_{i}(L)$ y $\beta(L)$ son los polinomios de rezagos en potencias no negativas de $L$.

Suponen que los polinomios de rezago $\lambda_{i}(L)$ y $\beta(L)$ son de orden finito no mayor a $q$, esto es, $\lambda_{i}(L)=\sum_{j=0}^{q} \lambda_{i j} L^{j}$ y $\beta(L)=\sum_{j=0}^{q} \beta_{j} L^{j}$. Producto de este supuesto, Stock y Watson demostraron que el modelo se puede reescribir como un modelo de factores estático, como se presenta a continuación, y que los factores $f$ pueden ser estimados utilizando la metodología de componentes principales.

$$
\begin{gathered}
y_{t+1}=\beta^{\prime} F_{t}+\gamma(L) y_{t}+\varepsilon_{t+1} \\
X_{t}=\Lambda F_{t}+e_{t}
\end{gathered}
$$

donde $F_{t}=\left(f_{t, \ldots,}^{\prime}, f_{t-q}^{\prime}\right)^{\prime}$ es de $r \times 1$.

La proyección de la variable para $h$ períodos adelante, $y_{t+h}$, se realiza en dos etapas. En un primer paso, a partir de la muestra $\left\{X_{t}\right\}_{t=1}^{T}$, se estiman los factores $\hat{F}$. En un segundo paso, se utilizan los factores y la variable a proyectar rezagada para obtener la proyección a partir de la ecuación

$$
\widehat{y_{t+h}}=\widehat{\alpha_{h}}+\widehat{\beta_{h}}(L) \widehat{F_{t}}+\widehat{\gamma_{h}}(L) y_{t}
$$

Otro de los modelos pioneros en la literatura de Nowcasting y usado como base para otras extensiones es el desarrollado por Giannone, Reichlin and Small (2008). Presentan un modelo de factores con frecuencias mixtas, cuyo objetivo es utilizar la información contenida en un conjunto amplio de series mensuales para pronosticar una variable trimestral.

La idea fundamental del enfoque es explotar la colinealidad de las series, resumiendo la información en un número reducido de factores comunes. Así, debido a la colinealidad, una proyección basada en los factores comunes es capaz de capturar la interacción dinámica de las series y de proveer un modelo parsimonioso para el pronóstico que requiere estimar sólo un número reducido de parámetros.

En particular, el modelo de factores dinámico (en su notación matricial) está dado por:

$$
x_{t m}=\mu+\Lambda f_{t m}+\xi_{t m}
$$


donde $x_{t m}$ es el vector de los $n$ indicadores mensuales estacionarios, $\mu$ es un vector de constantes, $f_{t m}$ es un vector de $r$ factores inobservables, $\Lambda$ es la matriz de factor loadings de dimensión $n \times r$, con $r<n$, y $\xi_{t m}$ es el componente idiosincrático, que se asume ruido blanco multivariado con una matriz de covarianza diagonal y ortogonales con los shock de los factores.

Para la estimación y para trabajar con el hecho de la falta de observaciones al final de la muestra ("ragged-edge"), en el trabajo se combina la metodología de componentes principales con el filtro de Kalman, en dos etapas. Primero, mediante componentes principales se calculan los factores que subyacen a los indicadores económicos mensuales contenidos en una versión balanceada de la base de datos, truncando la muestra hasta el momento que se cuenta con todas las observaciones.

En el segundo paso, el filtro de Kalman es utilizado para actualizar los factores, esto es, las estimaciones de las señales contenidas en las variables, utilizando la base de datos desbalanceada. Para ello es necesario tener definida la ecuación de movimiento de los factores, que se supone tienen un comportamiento autorregresivo de la forma:

$$
f_{t m}=\sum_{i=1}^{p} A_{i} f_{t m-i}+B \eta_{t m}
$$

donde $A$ es una matriz de $r \times r, B$ es una matriz de $r \times q$ y $\eta$ es el vector de dimensión $q$ de los shocks ruido blanco, con r mayor a $\mathrm{q}$.

Una vez que se cuenta con la estimación de los factores, el Nowcast del PIB puede obtenerse mediante una regresión por $\mathrm{MCO}$, en que se estiman los parámetros $\alpha$ y $\beta$ de la ecuación:

$$
\widehat{y_{t q}}=\alpha+\beta \widehat{f_{t q}}
$$

donde $\widehat{f_{t q}}$ es la agregación trimestral correspondiente a $\widehat{f_{t m}}$. De este modo, el pronóstico es definido como la proyección de la variable trimestral basada en los factores estimados de frecuencia trimestral.

Al observarse esta última ecuación puede apreciarse que se asemeja a la ecuación presentada en la metodología de bridge equations. Por eso, este enfoque puede interpretarse como un modelo bridge ampliado, que hace uso de un amplio número de 
variables y que hace un puente entre las variables de frecuencia mensual con el pronóstico de una variable de frecuencia trimestral.

Este modelo fue implementado inicialmente en el Board of Governors of the Federal Reserve para generar un Nowcast del PIB para la economía de Estados Unidos, producto de un proyecto iniciado en 2003. A partir de ese momento, varias versiones se han desarrollado en diferentes economías y se ha implementado en otros bancos centrales, como el Banco Central Europeo, y en organismos multilaterales, como el Fondo Monetario Internacional (Matheson, 2011).

También hay diversos estudios que aplican esta metodología para Estados Unidos (Lahiri and Monokroussos, 2011), para la zona del euro en su conjunto (Angelini et al., 2011; Banbura and Modugno, 2010; Banbura and Rünstler, 2011; Camacho and Perez-Quiros, 2009), para países integrantes de la zona del euro como Francia (Barhoumi et al., 2010), para Alemania (Schumacher and Breitung, 2008), Irlanda (D'Agostino et al., 2008), Holanda (de Winter, 2011), entre otros.

\section{$\underline{\text { 3. El Ejercicio de Nowcast }}$}

\subsection{Motivación}

El objetivo del presente trabajo consiste en realizar estimaciones tempranas del crecimiento de la Inversión a partir de un conjunto amplio de indicadores de alta frecuencia.

Conocer el desempeño de la Inversión es fundamental para los diversos agentes económicos para la toma de decisiones óptimas y es de gran relevancia para los hacedores de política para la implementación oportuna de políticas de incentivo a la Inversión y de políticas contracíclicas. En Argentina la Inversión representa aproximadamente entre el $15 \%$ y el 19\% del PIB, dependiendo del período considerado, ubicándose por debajo de los ratios de los países desarrollados o de los que presentan altas tasas de expansión económica. De este modo, es indispensable conocer el desempeño de la Inversión para favorecer a una asignación eficiente de los recursos y que permita lograr un crecimiento de la economía sostenible a largo plazo. 
En Argentina el Instituto Nacional de Estadísticas y Censos (INDEC) difunde la información de la Inversión en conjunto con la información del PIB en cuentas nacionales, que es una información con periodicidad trimestral y que se publica con rezago. El dato del PIB y del componente de Inversión se conocen recién a las 10 semanas de finalizado el trimestre. Por caso, los datos relativos al primer trimestre de 2017 se dieron a conocer en la tercera semana de junio de ese año. A su vez, tampoco publica el INDEC ningún indicador parcial adelantado de la Inversión que nos permita tener una idea inmediata de su comportamiento, tal como ocurre con el estimador mensual industrial (EMI), el indicador de actividad de la construcción (ISAC), las ventas minoristas, el estimador mensual de la actividad económica (EMAE), entre otros.

Por otro lado, está disponible un conjunto amplio de indicadores económicos de alta frecuencia que puede servir para obtener información valiosa sobre el ciclo de la Inversión. Esto es, indicadores de periodicidad mensual, semanal o diaria.

La metodología de Nowcasting, que implica realizar estimaciones de variables de baja frecuencia con indicadores de alta frecuencia, demostró ser capaz de predecir de manera eficiente los acontecimientos en el corto plazo y de superar en capacidad predictiva a los modelos univariados, particularmente en contextos de alta volatilidad. Entre las ventajas que presenta esta metodología mencionadas en la literatura (Giannone et al., 2008) es que permite utilizar una gran cantidad de series de distinta fuente, que las estimaciones son actualizadas a medida que la nueva información está disponible — de acuerdo con el calendario de actualización de las series - y que sirve para articular datos de alta frecuencia con datos de baja frecuencia.

\subsection{Datos}

La variable objetivo de la estimación es la Formación Bruta de Capital Físico (FBCF), intentando proyectar su crecimiento trimestral desestacionalizado. En este proceso se consideró inicialmente un conjunto amplio de indicadores económicos, 116 en total, a ser potencialmente utilizados. Entre ellos se incluyeron indicadores "duros" (hard indicators), clasificados como tales por transmitir señales bastante precisas sobre el desempeño de la economía — producción en la industrial, actividad en la construcción, actividad en el sector agropecuario, comercio, empleo, comercio exterior, variables 
fiscales y de actividad en Brasil — así como indicadores "blandos" (soft indicators), considerados menos precisos pero con información valiosa sobre las percepciones de distintos agentes económicos respecto de las condiciones presentes y futuras de la economía - encuestas de expectativas de empleo, confianza del consumidor, entre otros-. Generalmente hay sustanciales diferencias entre las series en cuanto a su publicación. En este sentido, las variables monetarias y financieras y las encuestas (indicadores blandos) están disponibles al final del período de referencia o aún con anterioridad (tal es el caso de la confianza del consumidor), mientras que los otros indicadores de la actividad económica (indicadores duros) se publican con rezago, que puede alcanzar a los 2 o 3 meses. De este modo, habría un trade-off entre la oportunidad y precisión de la información, por lo que la base de datos fue construida con la intención de contener ambos tipos de indicadores.

Para trabajar con las series se procedió en una primera instancia a realizar un estudio de la estacionalidad de las series mediante el programa ARIMA X12, realizándose el ajuste necesario en caso de corresponder, para obtener series sin estacionalidad. Luego se realizó la transformación logarítmica de las series, y mediante los test Dickey-Fuller aumentado y Phillips-Perron se analizó la existencia de raíz unitaria y, de corresponder, la fuente de no estacionariedad, con el fin de hacer la correcta transformación para obtener series estacionarias. Las tablas con los indicadores considerados y con los resultados del análisis de estacionalidad y estacionariedad se presentan en el Anexo I.

Tal como es usual en la realización de pronósticos macroeconómicos surge también en este trabajo el problema de seleccionar los indicadores a utilizar en la estimación dentro de un amplio conjunto de indicadores disponibles. Para seleccionar las series a incluir en el Nowcast se utilizó el coeficiente de correlación, siendo el criterio de elección inicial la existencia de una correlación contemporánea significativa entre la tasa de crecimiento de cada una de las variables y la tasa de crecimiento de la Inversión (FBCF). Para ello, al realizar la correlación de Spearman, se consideró al estadístico t del coeficiente de correlación, quedando seleccionadas aquellas series con un $t>1,96$ (nivel de confianza del 95\%) del conjunto potencial inicial (ver Anexo II). Luego, se conformaron tres grupos de indicadores para realizar el ejercicio de Nowcast con cada uno de ellos. Un primer grupo más amplio, Grupo 1 (G1), que comprende las series que presentan un valor del coeficiente de correlación superior a 0,30. Como resultado quedaron seleccionadas 36 series. El Grupo 2 (G2), formado por 26 series, resultado de tomar como criterio de 
selección un coeficiente de correlación superior a 0.35, y el Grupo 3 (G3), integrado por 19 series, que surge de considerar un coeficiente de correlación superior a 0.40 . Las series que incluye cada grupo se presenta en la Figura 1.

\section{Figura 1: Series Seleccionadas para la Estimación del Nowcast}

\begin{tabular}{|c|c|c|c|c|}
\hline Serie & Fuente & Grupo 1 & Grupo 2 & Grupo 3 \\
\hline Importaciones en cantidades- Nivel General & INDEC & $\times$ & $x$ & $\times$ \\
\hline Importaciones en cantidades de Bienes intermedios & INDEC & $\times$ & $x$ & $\times$ \\
\hline Importaciones en cantidades de Piezas y accesorios & INDEC & $x$ & $x$ & $x$ \\
\hline Importaciones en cantidades de Bienes de consumo & INDEC & $\times$ & $x$ & $\times$ \\
\hline Indice de Confianza del Consumidor-Condiciones Presentes & UTDT & $x$ & $x$ & $x$ \\
\hline Ventas minoristas en Ferreterías & CAME & $\times$ & $x$ & $\times$ \\
\hline Ventas minoristas de Muebles de Oficina & CAME & $\times$ & $\times$ & $\times$ \\
\hline Indice de Confianza del Consumidor - Nivel General-Nacional & UTDT & $x$ & $x$ & $x$ \\
\hline Indice de Producción Industrial - Automóviles & FIEL & $\times$ & $x$ & $\times$ \\
\hline Ventas minoristas de Electrodomésticos & CAME & $\times$ & $\times$ & $\times$ \\
\hline Indice de Confianza del Consumidor -Situación Personal & UTDT & $\times$ & $\times$ & $\times$ \\
\hline Precios de las Exportaciones de Productos Primarios & INDEC & $\times$ & $\times$ & $\times$ \\
\hline Precios de las Exportaciones de MOI & INDEC & $\times$ & $x$ & $\times$ \\
\hline Producción Nacional de Vehículos & ADEFA & $\times$ & $x$ & $x$ \\
\hline Indice de Confianza del Consumidor - Capital Federal & UTDT & $\times$ & $x$ & $x$ \\
\hline Indice de Producción Industrial - Bienes de capital & FIEL & $x$ & $x$ & $x$ \\
\hline Producción industrial de Brasil & Banco Central de Brasil & $x$ & $x$ & $x$ \\
\hline Precios de las Exportaciones - Nivel general & INDEC & $x$ & $x$ & $\times$ \\
\hline M2* Amplio Privado Nominal & BCRA & $x$ & $x$ & $x$ \\
\hline Indice de Producción Industrial - Bienes de consumo durables & FIEL & $x$ & $x$ & \\
\hline Laminados en caliente Total no Planos & Cámara del Acero & $x$ & $x$ & \\
\hline Expectativa neta de empleo & Ministerio de Trabajo & $x$ & $x$ & \\
\hline Préstamos con Tarjetas & BCRA & $x$ & $x$ & \\
\hline Escrituras en Ciudad de Buenos Aires & Colegio de Escribanos & $x$ & $x$ & \\
\hline Importaciones en cantidades de vehículos & INDEC & $\times$ & $\times$ & \\
\hline Ventas de Vehículos Nacionales & ADEFA & $\times$ & $x$ & \\
\hline Laminados en Frío & Cámara del Acero & $x$ & & \\
\hline Laminados en caliente Planos & Cámara del Acero & $x$ & & \\
\hline Indicador de actividad de Brasil & Banco Central de Brasil & $\times$ & & \\
\hline Ventas minoristas de Materiales para la Construcción & CAME & $\times$ & & \\
\hline Empresas que realizaron búsqueda de personal & Ministerio de Trabajo & $\times$ & & \\
\hline Exportaciones en cantidades de MOI & INDEC & $\times$ & & \\
\hline Ventas minoristas de Materiales Eléctricos & CAME & $\times$ & & \\
\hline Recaudación Impuesto a las Ganancias & MECON & $x$ & & \\
\hline Indice de Confianza del Consumidor -Expectativas & UTDT & $\times$ & & \\
\hline Indice de Confianza del Consumidor-Durables e Inmuebles & UTDT & $x$ & & \\
\hline
\end{tabular}

La muestra utilizada para estimar los modelos abarca desde el primer trimestre de 2006 hasta el primer trimestre de 2016. Luego se realizaron ejercicios de Nowcast del crecimiento de la Inversión para el período comprendido por el primer trimestre de 2011 y el segundo trimestre de 2017 para cada uno de los modelos generados. 
El ejercicio es pseudo-real time, dado que se confeccionó una base de datos tratando de replicar la información que hubiera estado disponible en cada momento del tiempo, considerando para ello el calendario de publicación de cada serie. De este modo, se toman en cuenta los rezagos en la publicación, pero se ignoran las revisiones que pudieron haber sufrido los datos, tanto de la Inversión como de los indicadores que se utilizan para la estimación. Esto último podría implicar una sobreestimación del ajuste de los modelos de pronóstico. Sin embargo, se cancelarían los efectos de la revisión de los datos sobre el pronóstico, dado que el método estadístico utilizado tiende a reducir el ruido del proceso, al extraer factores de un conjunto amplio de indicadores económicos. En este sentido, Shumacher y Breitung (2008) utilizan datos real-time para Alemania y no encuentran ningún impacto claro de la revisión de los datos sobre los errores de pronóstico del modelo de factores.

Para realizar la estimación del Nowcast de la Inversión, se divide el conjunto de indicadores en dos grupos, de acuerdo con el momento de publicación de las series: los que están disponibles en la primera quincena y aquellos que se difunden en la segunda mitad del mes. De este modo, producto de esta agrupación de las series y dado que el Nowcast se actualiza secuencialmente cuando se dispone de nueva información, se generan 6 estimaciones de la Inversión para cada trimestre. A modo de ejemplo, se presenta la actualización secuencial en la Figura 2.

Figura 2: Secuencia de Actualización del Nowcast

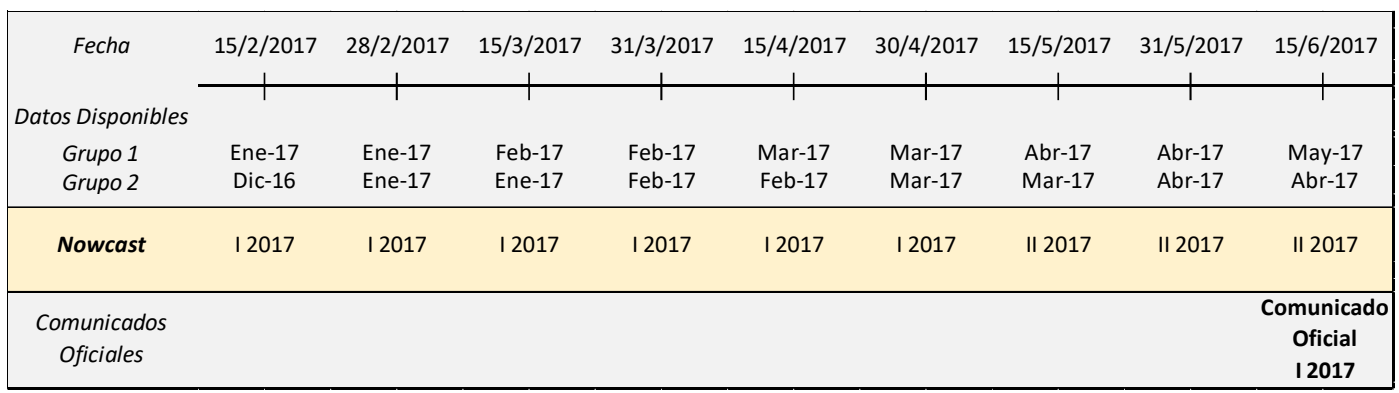




\subsection{Metodología de Estimación: Modelo de Factores Dinámicos}

La estimación del Nowcast de la Inversión se realiza considerando un modelo de factores, tal como es su aplicación generalizada para la estimación del PIB, en el que se relaciona una variable a pronosticar $y_{t+1}$ con un conjunto de predictores contenidos en el vector $X_{i t}$. El ejercicio de pronóstico es realizado en un proceso de dos pasos: primero se estiman los factores comunes al conjunto de los indicadores $X_{i t}$ por componentes principales, y posteriormente se utilizan esos factores para estimar $y_{t+1}$. La idea que subyace a esta metodología de modelos dinámicos de factores es que las variables en el conjunto de interés son impulsadas por un conjunto reducido de factores no observables. Así, la información contenida en un amplio número de predictores puede ser reemplazada por un número manejable de factores estimados.

Concretamente, la covarianza entre un gran número de $n$ series de tiempo, con sus adelantos y rezagos, puede ser representada por un número reducido de $q$ factores no observados, $\operatorname{con} n>q$.

Por lo tanto, dado un vector $X_{i t}$ de $n$ series de tiempo mensuales estacionarias $x_{t}=$ $\left(x_{1 t}, x_{2 t}, \ldots, x_{n t}\right)^{\prime}$, con $t=1, \ldots, T$, el vector de $n$ variables observables en el ciclo puede ser explicado por los rezagos distribuidos de $q$ factores comunes latentes más $n$ perturbaciones idiosincráticas que pueden eventualmente estar correlacionadas serialmente, así como correlacionadas entre ellas.

$$
X_{i t}=\lambda_{i}(L)^{\prime} f_{t}+\mu_{i t}
$$

Donde $f_{t}$ es un vector $q \times 1$ de factores no observables, $\lambda_{i}$ es un vector de polinomios de rezagos denominados dynamic factor loadings de dimensión $q \times 1$ y $\mu_{i t}$ son las perturbaciones idiosincráticas. Se supone que los factores y las perturbaciones idiosincráticas no están correlacionadas entre sí (ni en sus adelantos ni en sus rezagos), es decir $E\left(f_{t} \mu_{i t}\right)=0 \forall i, t$.

El objetivo es entonces estimar $E\left(y_{t} / X_{t}\right)$, modelando $y_{t}$ según:

$$
y_{t}=\beta(L)^{\prime} f_{t}+\gamma(L)^{\prime} y_{t-1}+\varepsilon_{t}
$$


Si los polinomios de rezagos $\lambda_{i}(L)$ y $\beta(L)$ son de orden finito $p$, Stock y Watson (2002) demostraron que los factores $f$ pueden ser estimados utilizando la metodología de componentes principales.

Si se define a la Inversión trimestral como el promedio de las observaciones mensuales latentes, $y_{t}^{Q}=\left(y_{t}+y_{t-1}+y_{t-2}\right) / 3$, y si se estiman los factores trimestrales $f_{t}^{Q}$ de los datos considerados como predictores, es posible obtener estimaciones tempranas de la Inversión, esto es, dentro del trimestre, utilizando la siguiente ecuación:

$$
{\widehat{y_{t}}}^{Q}=\beta(L)^{\prime} f_{t}^{Q}+\gamma(L)^{\prime} y_{t-1}^{Q}
$$

El ejercicio de Nowcast consistió en una primera instancia, como se comentó anteriormente, en seleccionar los indicadores a considerar en la estimación de acuerdo con el coeficiente de correlación de las series estacionarias y de la Inversión, también con su transformación a estacionaria. En base a esto se armaron 3 grupos de indicadores (G1, G2 y G3) a partir de los cuales se estimaron, mediante la metodología de componentes principales, los factores comunes a los indicadores pertenecientes a cada uno de estos grupos.

Para seleccionar el número de factores a considerar en la proyección, se utilizó el scree plot. De acuerdo con este gráfico, en que se dibujan los autovalores - que surgen del método de cálculo-, el criterio consiste en seleccionar la cantidad de factores que se suceden en la gráfica hasta que en la misma se produce un cambio en la pendiente o cuando la misma parece nivelarse con la parte derecha del dibujo. Teniendo en cuenta esto, se seleccionaron los primeros 3 o 4 factores, dependiendo del conjunto de información considerado (G1, G2 o G3), los que permiten explicar entre el 60\% y 77\% de la covarianza de las series de tiempo consideradas en cada conjunto para la estimación (ver Figura 3 y Anexo III). 
Figura 3: Scree Plot

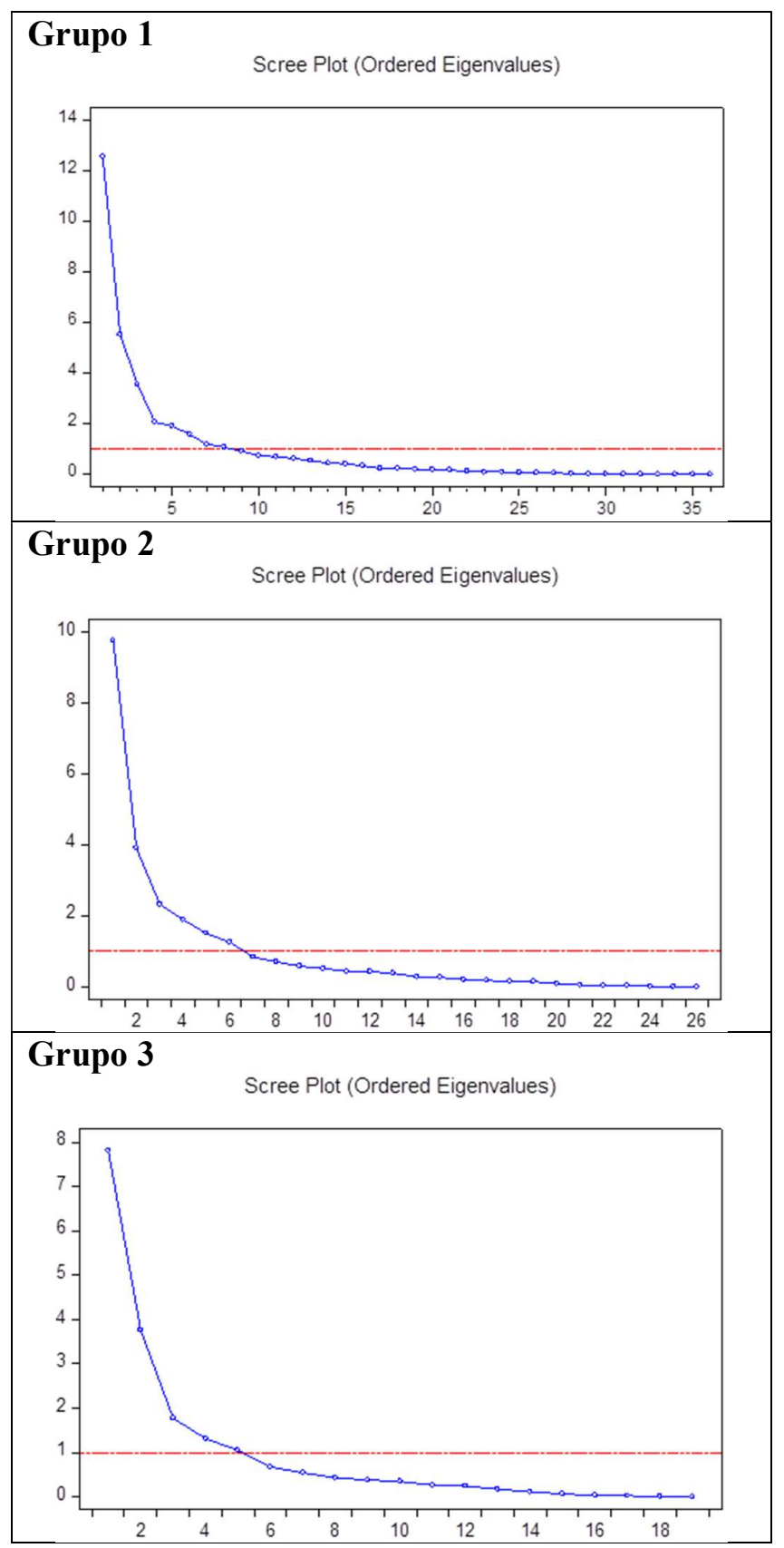

Los factores calculados se utilizaron para realizar la estimación de la Inversión. Se busca el modelo lo más parsimonioso posible, por lo que resultó significativo sólo el primer factor (ver Anexo IV). Este resultado está en línea con Stock y Watson (2002), que encuentran que sólo uno o dos factores son suficientes para obtener buenos pronósticos cuando se trata de una variable real. 
En todos los casos, los modelos fueron especificados para garantizar residuos ruido blanco, homocedásticos y normales.

El ejercicio de Nowcast se realizó mediante ventanas móviles (rolling window) con un tamaño de la ventana de estimación de 40 trimestres. De este modo, el tamaño de la muestra permaneció constante durante todo el ejercicio, al incorporarse la nueva información y descartarse la información más antigua. Como se mencionó, producto de la actualización de los datos, se obtuvieron 6 estimaciones para cada trimestre.

La elección de la metodología de factores radica en las ventajas que presenta para trabajar con un gran número de indicadores y en la capacidad para incorporar información de manera inmediata. De este modo, logra capturar los posibles quiebres de tendencia y mantiene sus cualidades en capacidad predictiva en períodos inestables o de alta volatilidad. Esta característica es de particular relevancia en el caso de Argentina, más aún al considerar el cambio de régimen evidenciado en los últimos años.

De manera contraria, la metodología de bridge equations utiliza generalmente modelos con series de datos de largo historial, con un número de indicadores relativamente más reducido, y que no siempre logran responder rápidamente a la nueva información y capturar un cambio de tendencia de la variable estimada. A su vez, como estos modelos habitualmente incorporan a las variables dependientes e independientes de manera rezagada, pueden tener una fuerte dependencia de los valores pasados de estas variables, lo que puede afectar a la capacidad predictiva en los períodos de alta volatilidad.

\subsection{Resultados de la Estimación}

En esta sección se presentan los resultados del ejercicio de Nowcast de la Inversión a partir de los tres modelos de factores especificados (Nowcast G1, Nowcast G2 y Nowcast G3), que surgen de considerar los tres conjuntos de información empleados (Grupo 1, Grupo 2 y Grupo 3), desde el primer trimestre de 2011 al segundo trimestre de 2017. También se presentan los resultados de la estimación, para igual período, provenientes de un modelo AR(1) para la Inversión, el que se considera como modelo benchmark contra el cual contrastar los resultados. 
Los resultados que surgen de la estimación realizada están libres del juicio de experto. La metodología utilizada para realizar el ejercicio de Nowcast está basada en un modelo estadístico que se actualiza cuando se cuenta con nueva información, de acuerdo con el calendario de publicación previsto, sin ninguna intervención del juicio de experto. Esto difiere, por ejemplo, del Nowcast del PIB que realiza el Banco de Inglaterra (BoE) y que utiliza el Comité de Política Monetaria como insumo para la toma de decisiones, en el que la estimación no es un resultado mecánico de un modelo (Anesti et. al, 2017). En particular, el Nowcast del PIB del BoE está conformado por los resultados de tres modelos (modelo industrial, modelo MIDAS-combinado y modelo de factores dinámicos), que son combinados o ponderados de acuerdo con el criterio del staff del banco y considerando a su vez otra información que los modelos pueden no haber sido capaces de capturar.

\section{Figura 4: Resultados de la Estimación de la Inversión: Nowcast vs. AR}

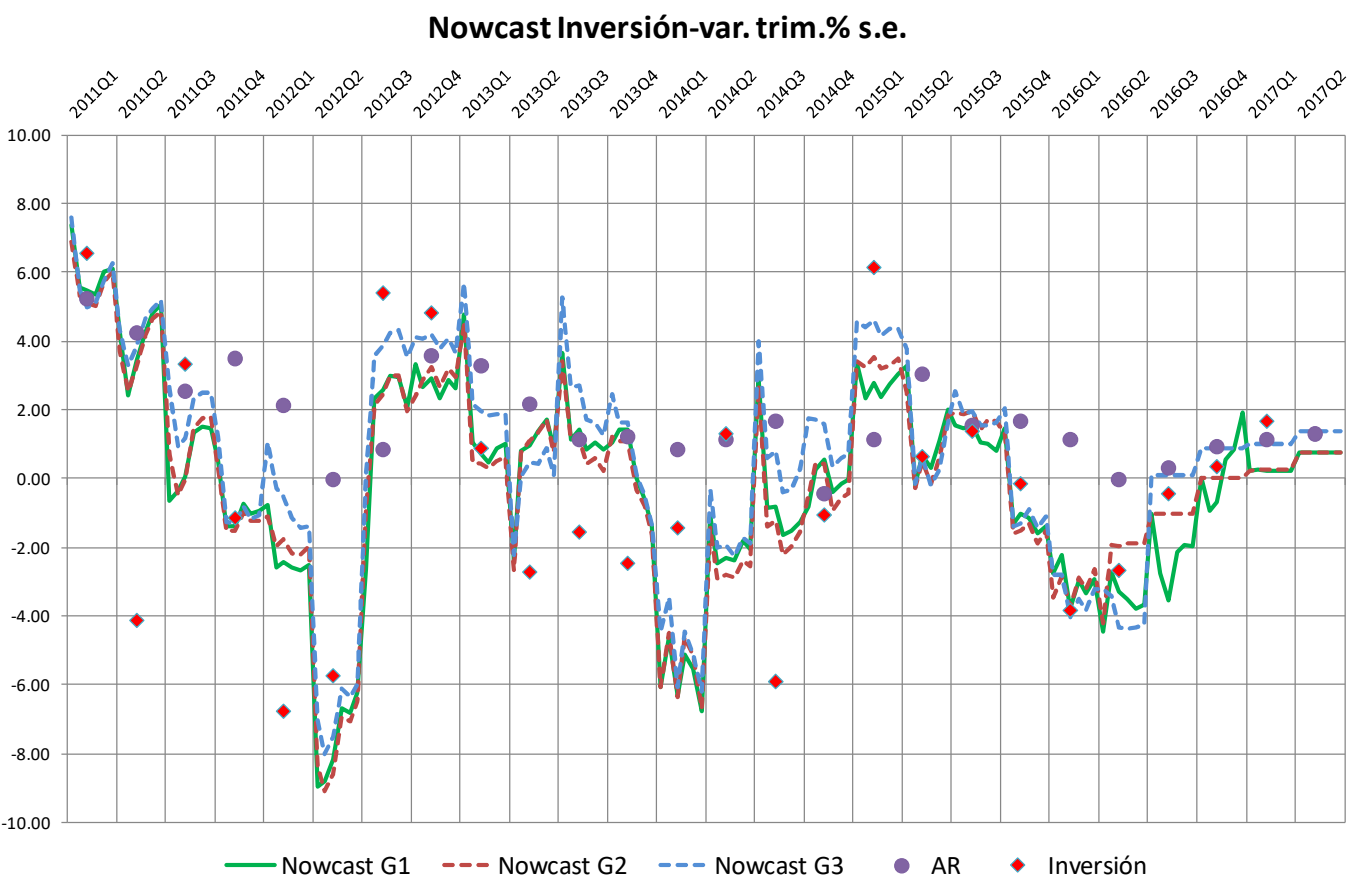

Según se desprende de la Figura 4, en la mayoría de los trimestres los modelos de factores (Nowcast G1, Nowcast G2 y Nowcast G3) arrojan un mejor pronóstico de la Inversión que el que surge del modelo AR considerado como benchmark. Esto se observa particularmente en los momentos en que hay un cambio en la dinámica de la Inversión 
entre los trimestres, en que los modelos de factores parecen poder reaccionar más rápidamente y reflejar el cambio de tendencia, producto de la incorporación inmediata de la nueva información (news).

Para evaluar la capacidad predictiva de los modelos se puede utilizar la raíz cuadrada del error cuadrático medio (RMSE, de acuerdo con su sigla en inglés) que surge de comparar los pronósticos y la realización de la variación trimestral de la Inversión para cada trimestre (valor observado). A partir de la observación de la Figura 5 que presenta la evolución de los RMSE para cada modelo en cada una de las seis realizaciones para el período de evaluación, no se puede distinguir una superioridad en capacidad predictiva de alguno de los modelos, si bien en términos generales los modelos de factores (Nowcast G1, Nowcast G2 y Nowcast G3) parecen presentar un menor RMSE que el exhibido por el modelo AR. Teniendo en cuenta este resultado, se abordaron distintas estrategias, a partir de los RMSE, para poder obtener alguna conclusión sobre la elección del modelo más adecuado para el Nowcast de Inversión.

Figura 5: Errores de Pronóstico: Nowcast vs. AR

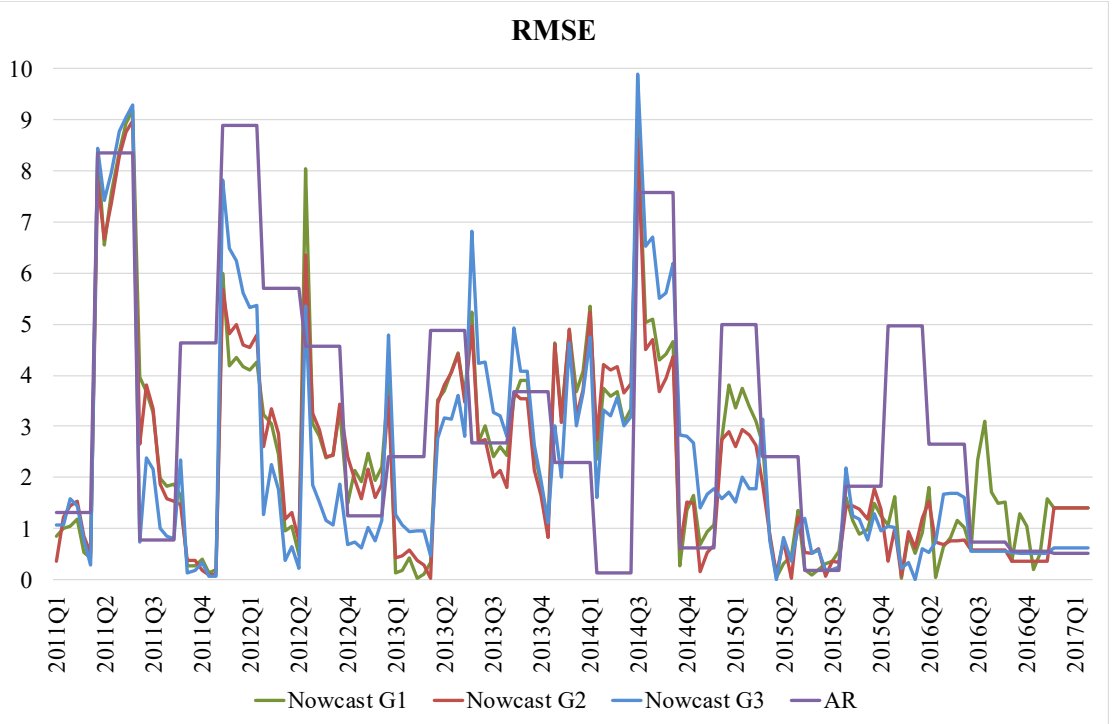

Una primera estrategia consistió en observar la distribución de los RMSE de cada uno de los modelos, reflejadas en un gráfico de caja o box plot. Este gráfico resume la información más relevante acerca de la distribución de una variable. La amplitud de la caja da una idea de la dispersión de los datos, dado que representa el rango intercuartílico 
(RIC) comprendido entre los percentiles 25 y 75 de la distribución y contienen el 50\% central de las observaciones. De este modo, cuanto más compacta sea la caja mayor es la concentración de la variable en torno al valor central de la distribución que es la mediana (la línea sólida que se observa al interior de la caja). Que esta línea se ubique por debajo de la media (representada en el gráfico por la cruz) es una evidencia de asimetría positiva en la distribución de los RMSE y significa que los valores más elevados traccionan a la media por encima de la mediana. Los "bigotes" se extienden desde la mediana hasta $+/$ 1,5 del RIC y las observaciones por encima o por debajo de los "bigotes" son observaciones atípicas o outliers.

De acuerdo con la distribución de los RMSE, se podría concluir que los modelos de factores (Nowcast G1, Nowcast G2 y Nowcast G3) tienen una mejor performance que el modelo AR, ya que para los tres modelos de factores la caja es más compacta, y la mediana y la media son inferiores a las del modelo AR, más allá de los valores extremos o outliers que presentan. No obstante, es difícil establecer cuál de los modelos de factores posee la mejor performance, si bien podría pensarse a priori que correspondería a alguno de los modelos Nowcast G2 o Nowcast G3, pues exhiben los menores valores en media y mediana (ver Figura 6).

\section{Figura 6: Errores de Pronóstico: Nowcast vs. AR}

\section{Box Plot RMSE}

12
10
8
6
4
2
0

\begin{tabular}{|l|l|}
\hline & \\
8 & \\
6 & \\
4 & \\
2 & \\
\hline
\end{tabular}
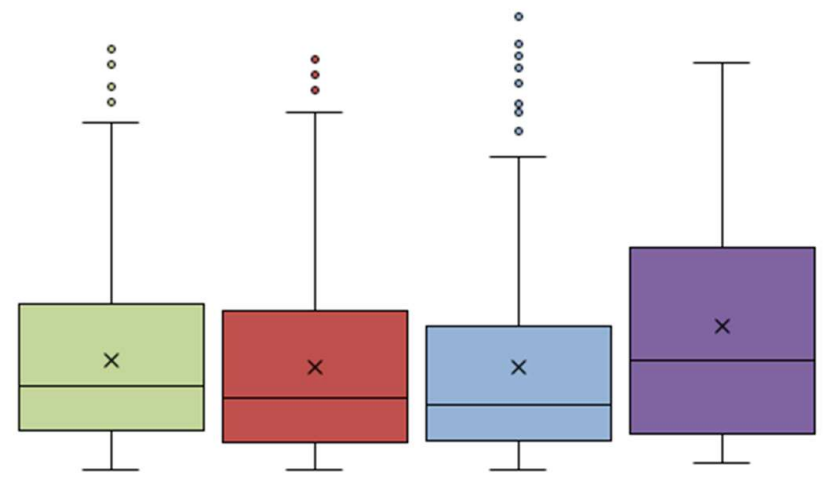

$\square$ Nowcast G1 $\square$ Nowcast G2 $\square$ Nowcast G3 $\square$ AR 
Otra estrategia para poder tener conocimiento sobre la performance de los modelos en cuanto a la capacidad predictiva consistió en analizar la cantidad de veces sobre el total (frecuencia) en la que cada modelo presenta el menor error de pronóstico, en términos de RMSE (ver Figura 7). Para esto se consideró la muestra completa (desde el primer trimestre de 2011 al primer trimestre de 2017) y una muestra más reciente, que también incluye al cambio estructural o cambio de régimen de diciembre de 2015 (desde el primer trimestre de 2015 al primer trimestre de 2017). De acuerdo con este criterio, el modelo Nowcast G3 es el que presenta la mejor performance en la muestra completa, tanto en relación con el AR como con respecto al resto de los modelos de factores (Nowcast G1 y Nowcast G2). Para la muestra más reciente, el modelo Nowcast G3 también exhibe el mejor desempeño (supera al AR y a los modelos Nowcast G1 y Nowcast G2).

Figura 7: Desempeño en Pronóstico: Nowcast vs. AR

\begin{tabular}{|c|c|c|c|c|}
\hline \multicolumn{5}{|c|}{$\begin{array}{l}\text { Frecuencia con la cual el modelo está rankeado en primer lugar en } \\
\text { cuanto a capacidad predictiva (menor RMSE, en \%) }\end{array}$} \\
\hline & $\begin{array}{l}\text { Nowcast } \\
\text { Grupo } 1\end{array}$ & $\begin{array}{l}\text { Nowcast } \\
\text { Grupo } 2 \\
\end{array}$ & $\begin{array}{c}\text { Nowcast } \\
\text { Grupo } 3\end{array}$ & $A R$ \\
\hline $\begin{array}{l}\text { muestra completa } \\
2011 q 1-2017 q 1\end{array}$ & 0.167 & 0.247 & 0.340 & 0.247 \\
\hline $\begin{array}{l}\text { muestra } \\
\text { 2015q1-2017q1 }\end{array}$ & 0.148 & 0.259 & 0.407 & 0.185 \\
\hline
\end{tabular}

De este modo, parece que la inclusión de un mayor número de indicadores no estaría mejorando la capacidad de pronóstico del modelo, dado que el modelo Nowcast G3 es el que incluye la menor cantidad de indicadores (Grupo 3 conformado por 19 series). Estos resultados también son reflejados por la literatura empírica. En este sentido, Banbura y Mondungo (2010) encuentran en su estimación que los modelos de factores superan en capacidad predictiva al benchmark AR, lo que confirma la importancia de contar con información contemporánea contenida en los indicadores mensuales. Sin embargo, entre los diversos modelos de factores que contienen distinta cantidad de información, encuentran que el que presenta la mayor desagregación de la información no arroja los mejores resultados de pronósticos y es superado por las estimaciones que consideran una desagregación mediana o pequeña. De acuerdo con sus conclusiones, esta situación 
podría responder a que se genera una dificultad en extraer las señales relevantes. En igual dirección están los resultados encontrados por Brave et. al. (2016), quienes al realizar una estimación para el PIB de Estados Unidos concluyen que incluir mayor cantidad de variables o predictores no necesariamente aumenta la capacidad predictiva del modelo.

\section{$\underline{\text { 4. Evaluación de Capacidad Predictiva }}$}

Para evaluar si las diferencias en capacidad predictiva encontradas en la sección anterior son estadísticamente significativas y seleccionar el modelo que presenta la mejor performance de pronóstico se utiliza el test de Giacomini y White (2003).

Como se mencionó, conocer el desempeño y el pronóstico de las principales variables económicas es de fundamental relevancia para los agentes económicos y, en este contexto, uno de los problemas a enfrentar es seleccionar el mejor método de pronóstico entre dos o más alternativas. La respuesta econométrica a este problema consiste en el desarrollo de tests que permitan comparar la habilidad predictiva de dos métodos de pronósticos alternativos, dada la función de pérdida.

Giacomini y White (2003) propusieron un test para la selección de pronósticos real-time, esto es, para establecer cuál entre dos métodos competitivos de pronóstico es el que generará un mejor pronóstico en el futuro más próximo.

El enfoque metodológico planteado por Giacomini y White se basa en expectativas condicionales. De acuerdo con el trabajo, el enfoque consiste en evaluar lo que ellos llaman el método de pronóstico, lo que incluye al modelo así como también las decisiones que se deben tomar en cuanto a la elección de los procedimientos y de los datos a incluir, dado que todos los elementos afectan la performance del pronóstico. En este sentido, señalan que el hecho de tener un modelo que aproxime correctamente el proceso generador de los datos no garantiza que genere buenos pronósticos. Así, establecen que esta metodología es relevante cuando el objetivo es encontrar modelos con buena capacidad predictiva en lugar de evaluar la validez de algún modelo.

Por otro lado, una de las ventajas que presenta esta metodología es que permite trabajar con datos heterogéneos, en el sentido que las series de interés pueden presentar un proceso generador de los datos variable en el tiempo — no es necesario contar sólo con series que 
presenten un proceso estacionario- Según esgrimen, varias pueden ser las razones por las cuales las series económicas utilizadas para realizar los pronósticos pueden presentar heterogeneidades a través del tiempo. Ellas abarcan desde modificaciones en la medición, en el relevamiento, en la definición de la construcción de las variables, hasta cambios de los responsables de las instituciones y de la confección de los estadísticos. De este modo, el hecho de contar con heterogeneidad en la información también afecta al método de estimación, haciendo necesario contar con estimaciones de memoria finita. En este sentido, en vez de utilizar un enfoque recursivo, en el que se expande la ventana de los datos para la estimación adicionando la nueva información, se utiliza un procedimiento de ventanas móviles (rolling window), en el que los pronósticos están basados en una ventana móvil de los datos en que se descartan las observaciones más antiguas. En este enfoque, la ventana de estimación se considera como uno de los elementos de elección del método de pronóstico bajo evaluación.

Otra ventaja que presenta el test es que refleja el efecto de la incertidumbre en la estimación sobre la performance relativa de pronóstico. Al expresar la hipótesis nula en términos del parámetro estimado y al considerar una ventana de estimación finita, se genera una estimación que presenta una incertidumbre que no se reduce asintóticamente, sino que permanece como un factor determinante del pronóstico y por lo tanto de su performance.

Adicionalmente, el test permite comparar de manera unificada pronósticos provenientes de una amplia variedad de modelos, entre los que se incluyen modelos anidados y no anidados. También se puede utilizar para comparar la performance de pronósticos utilizando distintas técnicas de estimación, como métodos bayesianos, semi-paramétricos o no paramétricos.

A su vez, también presenta una ventaja práctica, que radica en la facilidad de su computo a través de la realización de una regresión en los paquetes econométricos tradicionales.

Específicamente, el test consiste en comparar dos modelos alternativos de pronóstico, para la variable de interés en $\tau$ pasos adelante, esto es $Y_{t+\tau}$. Los pronósticos son formulados en el momento $t$ y se basan en el conjunto de información disponible $\mathcal{F}_{t}$. Los dos pronósticos que se comparan pueden definirse como $\hat{f}_{m, t}$ y $\hat{g}_{m, t}$, donde $f$ y $g$ son funciones mensurables. Los subíndices implican que el pronóstico realizado en el 
momento $t$ es una función mensurable de la muestra de, como máximo, tamaño $m$, considerando las más recientes $m$ observaciones.

Los estimadores pueden ser paramétricos, semi-paramétricos o no paramétricos. El único requisito es que $m$ (el tamaño de la ventana de estimación) debe ser finito. Todo el resto de los elementos de la estimación, esto es el modelo, el procedimiento de estimación, el tamaño de la ventana de estimación y la función de pérdida, son tratados como parte de la elección que realiza quien hace el pronóstico y parte de lo que se llama el método de estimación que está bajo evaluación.

La evaluación es realizada en una simulación out-of-sample. Se considera una muestra de tamaño $T$. Como los datos indexados $1, \ldots, m$ son usados para la estimación del primer conjunto de parámetros, el primer pronóstico $\tau$ pasos adelante es formulado al momento $m$ y comparado con la realización $Y_{m+\tau}$. El segundo pronóstico es producido al mover la ventana de estimación un paso hacia adelante y estimar los parámetros sobre los datos indexados $2, \ldots, m+1$. Este pronóstico es comparado con la realización $Y_{m+1+\tau}$. Este procedimiento ese iterado y el último pronóstico es generado en el momento $T-\tau$, estimando los parámetros sobre los datos indexados $T-\tau-m+1, \ldots, T-\tau$, y es comparado con la realización $y_{T}$. Este procedimiento de ventanas móviles genera una secuencia de $n \equiv T-\tau-m+1$ pronósticos y de los respectivos errores de pronóstico.

La secuencia de los pronósticos out-of-sample así producidos son evaluados a partir de una función de pérdida $L_{t+\tau}\left(Y_{t+\tau}, \hat{f}_{m, t}\right)$, que depende del pronóstico y de la realización de la variable. Dada la función de pérdida, la hipótesis nula de igual capacidad predictiva condicional de los pronósticos $f$ y $g$ se puede escribir:

$$
H_{0}: E\left[L_{t+\tau}\left(Y_{t+\tau}, \hat{f}_{m, t}\right)-L_{t+\tau}\left(Y_{t+\tau}, \hat{g}_{m, t}\right) / \mathcal{F}_{t}\right]=0
$$

o alternativamente

$$
H_{0}: E\left[\Delta L_{m, t+\tau} / \mathcal{F}_{t}\right]=0, \text { con } t=1,2, \ldots
$$

Si la hipótesis nula de igual capacidad predictiva condicional de los métodos de pronóstico $f$ y $g$ es rechazada, se incrementa la posibilidad de que se pueda seleccionar en el momento $T$ el mejor método de pronóstico para el período $T+\tau$.

En la práctica, el test consiste en realizar una regresión de las diferencias en las funciones de pérdida contra una constante y evaluar su significatividad utilizando el estadístico $t$ 
convencional para la hipótesis nula de un coeficiente igual a 0 . En el caso en que el horizonte de pronóstico sea mayor a uno, los errores estándar se calculan utilizando el estimador de covarianzas de Newey-West, que permite la presencia de heteroscedasticidad y autocorrelación residual. En este caso, como es un pronóstico un período adelante, no es necesario realizar este ajuste.

Los resultados de usar el test de Giacomini y White para evaluar la capacidad predictiva del Nowcast a partir de los diversos modelos de factores (Nowcast G1, Nowcast G2 y Nowcast G3) en relación con el AR se muestran en la Figura 8.

Figura 8: Evaluación Capacidad Predictiva: Nowcast vs. AR

Resultados del Test Giacomini y White

\begin{tabular}{|c|c|c|c|}
\hline \multicolumn{4}{|c|}{ Muestra Q1 2011 - Q1 2017} \\
\hline & coeficiente & estadístico t & $p$-value \\
\hline Nowcast G1 vs AR & -6.51 & -4.75 & 0.0000 \\
\hline Nowcast G2 vs AR & -7.27 & -5.54 & 0.0000 \\
\hline Nowcast G3 vs AR & -6.18 & -5.17 & 0.0000 \\
\hline \multicolumn{4}{|c|}{ Muestra Q1 2015 - Q1 2017} \\
\hline & coeficiente & estadístico t & p-value \\
\hline Nowcast G1 vs AR & -4.71 & -4.16 & 0.0001 \\
\hline Nowcast $G 2$ vs $A R$ & -5.66 & -4.94 & 0.0000 \\
\hline Nowcast G3 vs AR & -6.08 & -4.82 & 0.0000 \\
\hline
\end{tabular}

Se puede observar que todos los modelos de factores superan al AR considerado como benchamark, siendo las diferencias estadísticamente significativas (p-value de 0.000). Esto es, al realizar el test de Giacomini y White, el coeficiente es negativo, indicando que los errores de pronóstico de los modelos de factores son inferiores a los errores de pronóstico del modelo $\mathrm{AR}$, y estadísticamente significativo, dado el valor del estadístico t y el $p$-value asociado. Este resultado se mantiene tanto para la muestra comprendida entre el primer trimestre de 2011 y el primer trimestre de 2017 como para muestra que se inicia en el primer trimestre de 2015 y finaliza el primer trimestre de 2017. Este resultado es interesante debido a que ambas muestras incluyen un punto de inflexión o cambio de 
régimen, en el que los modelos Nowcast responden más favorablemente que el modelo autoregresivo.

También se realizó el test de Giacomini y White para comparar la capacidad de pronóstico relativa de los diversos modelos de factores. Esto es, se evaluó la capacidad de pronóstico relativa de los modelos Nowcast realizados con los distintos grupos de indicadores (Grupo 1, Grupo 2 y Grupo 3), definidos anteriormente. Los resultados para la muestra más amplia indican que el Nowcast G2 supera en capacidad predictiva al Nowcast G1, dado el coeficiente negativo y estadísticamente significativo, y que también supera al Nowcast G3, a un nivel de significatividad del 10\%. En tanto, de la comparación del Nowcast G3 con el Nowcast G1 no se pueden sacar conclusiones, pues el coeficiente no es estadísticamente significativo.

Por su parte, si consideramos la muestra del período más reciente, entre el primer trimestre de 2015 y el primer trimestre de 2017, se puede concluir que el Nowcast G2 tiene una mejor capacidad de pronóstico que el Nowcast G1, dado un coeficiente negativo y estadísticamente significativo. En tanto, el Nowcast G3 presenta un mejor desempeño en pronóstico que el Nowcast G1 y, a un nivel de significativa del 10\%, también es superior en capacidad predictiva al Nowcast G2 (ver Figura 9).

De este modo, el modelo Nowcast G3, el que se genera con el grupo más reducido de indicadores (19 series), es el que presenta la mejor performance para el período más reciente y el que por lo tanto se selecciona para realizar las estimaciones del Nowcast entre los modelos aquí evaluados. 
Figura 9: Evaluación Capacidad Predictiva: Nowcast

Resultados del Test Giacomini y White

\begin{tabular}{|c|c|c|c|}
\hline \multicolumn{4}{|c|}{ Muestra Q1 2011 - Q1 2017} \\
\hline & coeficiente & estadístico $t$ & $\mathrm{p}$-value \\
\hline Nowcast $\mathrm{G} 2$ vs $\mathrm{G} 1$ & -0.76 & -3.02 & 0.0029 \\
\hline Nowcast $\mathrm{G} 2$ vs $\mathrm{G} 3$ & -1.10 & -1.96 & 0.0519 \\
\hline Nowcast G3 vs G1 & 0.34 & 0.57 & 0.5722 \\
\hline \multicolumn{4}{|c|}{ Muestra Q1 2015 - Q1 2017} \\
\hline & coeficiente & estadístico $t$ & $\mathrm{p}$-value \\
\hline Nowcast G2 vs G1 & -0.95 & -3.46 & 0.0011 \\
\hline Nowcast G3 vs G2 & -0.48 & -1.74 & 0.0874 \\
\hline Nowcast G3 vs G1 & -1.43 & -3.30 & 0.0017 \\
\hline
\end{tabular}

\section{Pooling de Pronósticos}

La literatura de pronóstico ha enfatizado que el pooling o la combinación de diferentes pronósticos puede resultar en una mejor performance en comparación con los modelos individuales. El pooling de pronósticos implica la combinación de dos o más pronósticos derivados de modelos que usan diferentes predictores para producir un pronóstico.

Teniendo en cuenta los resultados obtenidos en las secciones anteriores, se procedió a realizar un pooling de los pronósticos generados por los modelos Nowcast G2 y Nowcast G3, utilizando ponderadores $w_{i}$ de igual peso.

De acuerdo con Ahumada y Garegnani (2012), la utilización de un promedio simple parece ser un método útil para ponderar los pronósticos debido a su simplicidad y al hecho de que la ganancia de utilizar otros métodos no parece ser muy elevada.

Confeccionado el pooling de pronósticos y calculados los respectivos RMSE para cada una de las estimaciones generadas, se procedió a realizar el test del Giacomini y White para evaluar su capacidad predictiva relativa al resto de los modelos. Las muestras que se consideran para realizar el test son las mismas que las de la sección anterior.

De acuerdo con los resultados que se muestran en la Figura 10, se puede concluir que tanto para la muestra completa (desde el primer trimestre de 2011 hasta el primer trimestre 
de 2017) como para la muestra más reciente (desde el primer trimestre de 2015 hasta el primer trimestre de 2017) el pooling supera en capacidad predictiva al modelo AR, dado que el coeficiente es negativo y estadísticamente significativo.

Figura 10: Evaluación Capacidad Predictiva: Pooling de Pronósticos

\begin{tabular}{|c|c|c|c|}
\hline \multicolumn{4}{|c|}{ Resultados del Test Giacomini y White } \\
\hline \multicolumn{4}{|c|}{ Muestra Q1 2011 - Q1 2017} \\
\hline & coeficiente & estadístico t & $\mathrm{p}$-value \\
\hline Pooling vs AR & -7.01 & -5.72 & 0.0000 \\
\hline Pooling vs G1 & -0.49 & -1.38 & 0.1693 \\
\hline Pooling vs G2 & 0.26 & 0.97 & 0.3333 \\
\hline Pooling vs G3 & -0.83 & -2.87 & 0.0047 \\
\hline \multicolumn{4}{|c|}{ Muestra Q1 2015 - Q1 2017} \\
\hline & coeficiente & estadístico t & $\mathrm{p}$-value \\
\hline Pooling vs AR & -6.12 & -5.11 & 0.0000 \\
\hline Pooling vs G1 & -1.41 & -4.24 & 0.0001 \\
\hline Pooling vs G2 & -0.46 & -3.51 & 0.0009 \\
\hline Pooling vs G3 & -0.04 & -0.25 & 0.8026 \\
\hline
\end{tabular}

En cuanto a su comparación con el resto de los modelos, se puede observar que para la muestra completa el pooling de pronósticos (de Nowcast G2 y Nowcast G3) supera en capacidad predictiva al Nowcast G3 de manera individual, mientras que en relación con el Nowcast G1 y el Nowcast G2 no se pueden sacar conclusiones, pues los coeficientes no son estadísticamente significativos.

Para la muestra más reciente, los resultados de Giacomini y White señalan que el pooling de pronósticos (de Nowcast G2 y Nowcast G3) posee una mejor performance que el Nowcast G1 y el Nowcast G2, cada uno de ellos de manera individual. En tanto, no resulta superador al Nowcast G3, dado que el coeficiente no es estadísticamente significativo.

Estos resultados están en línea con los obtenidos en la sección anterior, en los que se encuentra que para la muestra completa el Nowcast G2 arroja los mejores resultados (el pooling exhibe un coeficiente positivo y no estadísticamente significativo), mientras que 
para la muestra reducida el Nowcast G3 es el que presenta la mejor capacidad de pronóstico relativa.

De todos modos, es importante enfatizar que los modelos Nowcast individuales y combinados son superiores al modelo AR para las muestras consideradas. Estos resultados ponen en evidencia la superioridad de los modelos Nowcast para la toma de decisiones y proyección de la Inversión. Dentro de estos modelos, para el período más reciente, el que incorpora la menor cantidad de indicadores es el que presenta el mejor desempeño, en línea con la literatura más reciente en que incluir mayor cantidad de variables o predictores no necesariamente aumenta la capacidad predictiva del modelo.

\section{$\underline{\text { 6. Conclusiones }}$}

Mientras que tener un conocimiento sobre la Inversión, así como del resto de las variables macroeconómicas, es fundamental para los agentes económicos y los hacedores de política, las cifras oficiales de Cuentas Nacionales poseen una frecuencia trimestral y se publican con rezago.

En el presente trabajo se realizó una estimación en tiempo real de la evolución de la Inversión a partir de un conjunto amplio de indicadores económicos de alta frecuencia, lo que se conoce en la literatura como Nowcasting. El Nowcasting - definido como la predicción del presente, el futuro muy cercano y el pasado muy reciente - ha demostrado ser una herramienta útil para superar el problema de contar con información válida a distintas frecuencias.

El ejercicio de Nowcast realizado permite contar con una primera estimación de la Inversión en el trimestre a 45 días de iniciado el mismo, con subsecuentes actualizaciones quincenales, lo que implica una ganancia informativa en cuanto a esta variable. De este modo, el Nowcast de Inversión constituye un elemento valioso para las decisiones de política.

Para realizar el Nowcast se consideraron tres grupos de indicadores de frecuencia mensual y mediante modelos de factores dinámicos (Nowcast G1-36 series_-, Nowcast G2 - 26 series-y Nowcast G3 -19 series-) se pronosticó el crecimiento trimestral de la Inversión. Luego, se estudiaron los errores de pronóstico y se utilizó el test de Giacomini 
y White para analizar la capacidad predictiva relativa de los modelos Nowcast y de un modelo AR(1) considerado como un benchmark.

De las evaluaciones realizadas se desprende que los modelos Nowcast superan en capacidad predictiva al modelo AR, tanto para una muestra que comprende entre el primer trimestre de 2011 y el primer trimestre de 2017 como para una muestra más reciente, que abarca desde primer trimestre de 2015 hasta el primer trimestre de 2017. Este resultado es interesante debido a que ambas muestras incluyen un punto de inflexión o cambio de régimen, y los modelos Nowcast responden más favorablemente que el modelo autoregresivo al incorporar de manera inmediata la nueva información. Estos resultados ponen en evidencia la superioridad de los modelos Nowcast para la toma de decisiones y proyección de la Inversión.

Adicionalmente, se puede concluir que el modelo de factores Nowcast G3 genera el mejor pronóstico de la Inversión y es el que evidencia la mejor capacidad predictiva en el período más reciente. De este modo, dado que el Grupo 3 es el que contiene menor cantidad de indicadores -19 series_-, el hecho de incluir un mayor número de indicadores no estaría mejorando la capacidad de pronóstico del modelo.

También se realizó un ejercicio de pooling de pronósticos con los modelos Nowcast G2 y Nowcast G3 - los que presentan el mejor desempeño relativo-. El test de Giacomini y White señala que la capacidad de pronóstico del pooling es superior a la del modelo AR (para ambas muestras consideradas). Para la muestra más reciente, el test indica que el pooling exhibe una mejor performance que los modelos Nowcast G1 y Nowcast G2, pero no se puede aseverar que sea superior a la del modelo Nowcast G3, por lo que este último sería el modelo elegido para realizar la estimación de la Inversión.

Cabe mencionar que el Nowcast de Inversión está basado en un modelo estadístico que se actualiza automáticamente, sin ninguna intervención del juicio de expertos. De este modo, permite a los hacedores de política tener un conocimiento en tiempo real de la Inversión, lo que es fundamental para la implementación oportuna de políticas de incentivo a la Inversión y de políticas contracíclicas. En este sentido, el Nowcast de Inversión es una herramienta que, al brindar información del desempeño de la Inversión, permite realizar una asignación eficiente de los recursos para lograr un crecimiento de la economía sostenible a largo plazo. 


\section{$\underline{\text { 7. Referencias Bibliográficas }}$}

Ahumada, Hildegar and Maria Lorena Garegnani. 2012. "Forecasting a monetary aggregate under instability: Argentina after 2001". International Journal of Forecasting, 28, p.p.412-427.

Anesti, Nikoleta, Simon Hayes, Andre Moreira and James Tasker. 2017. "Peering into the present: the Bank's approach to GDP nowcasting". Bank's Monetary Analysis Directorate. Bank of England. Quarterly Bulletin 2017 Q2.

Angelini, Elena, Gonzalo Camba-Mendez, Domenico Giannone, Lucrezia Reichlin and Gerhard Rünstler. 2011. "Short-term forecasts of euro area GDP growth". The Econometrics Journal, 14: C25-C44.

Baffigi Alberto, Roberto Golinelli and Giuseppe Parigi. 2004. "Bridge models to forecast the euro area GDP". International Journal of Forecasting. Vol. 20. Issue 3, p.p. 447-460.

Banbura, Marta, Domenico Giannone, and Lucrezia Reichlin. 2010. "Nowcasting". ECARES Working paper 2010-021.

Banbura, Marta, Domenico Giannone, Michel Mondungo and Lucrezia Reichlin. 2013. "NowCasting and the Real-Time Data Flow”. ECB Working Paper Series. № 1564.

Banbura, Marta, and Michel Mondungo. 2010. "Maximun Likelihood Estimation of Factor Models on Data Sets with Arbritrary Pattern of Missing Data". ECB Working Paper Series. $\mathrm{N}^{\circ}$ 1189.

Banbura, Marta and Gerhard Rünstler. 2011. "A look into the factor model black box: Publication lags and the role of hard and soft data in forecasting GDP". International Journal of Forecasting, 27(2), p.p. 333-346.

Barhoumi, Karim, Olivier Darné and Laurent Ferrara. 2010. ¿Are disaggregate data useful for factor analysis in forecasting French GDP? Journal of Forecasting, 29(1-2), p.p. 132-144.

Barhoumi, Karim, Olivier Darné, Laurent Ferrara and Bertrand Pluyaud. 2012. "Monthly GDP forecasting using bridge models: application for the French economy". Bulletin of Economic Research, 2012. Vol. 64. Issue Supplement 1, s53-s70.

Bencivelli, Lorenzo, Massimiliano Marcellino M. and Gianluca Moretti. 2012. "Selecting predictors by Bayesian model averaging in bridge models". Banca d'Italia. Working Paper 872. Rome.

Blanco, Emilio, Laura D'Amato, Fiorella Dogliolo, Lorena Garegnani. 2017. "Nowcasting GDP in Argentina: Comparing the predictive ability of different models".

Brave, Scott A., R. Andrew Butters and Alejandro Justiniano. 2016. "Forecasting Economic Activity with Mixed Frequency Bayesian VARs". Working Paper, No. 2016-05. Federeal Reserve Bank of Chicago.

Camacho, Máximo y Gabriel Perez-Quiros. 2009. "Ñ- STING: España Short Term Indicador of Growth". Documentos de Trabajo N 0912. Banco de España.

D'Agostino, Antonello, Kieran Mc Quinn and Derry O'Brien. 2008. "Now-casting Irish GDP." Research Technical Papers 9/RT/08. Central Bank \& Financial Services Authority of Ireland.

D'Amato, Laura, Lorena Garegnani y Emilio Blanco. 2015. "Nowcasting de PIB: evaluando las condiciones cíclicas de la economía argentina". Estudios BCRA. Documentos de trabajo 2015/69.

D'Amato, Laura, Lorena Garegnani y Emilio Blanco. 2011. "Using the Flow of High Frequency Information for Short Term Forecasting of Economic Activity in Argentina". Ensayos Económicos 64. BCRA.

De Winter, Jasper. (2011): "Forecasting GDP growth in times of crisis: private sector forecasts versus statistical models." DNB Working Papers 320, Netherlands Central Bank, Research Department.

Drechsel, Katja and Laurent Maurin. 2008. "Flow of Conjunctural Information and Forecast of Euro Area Economic Activity”. European Central Bank. Working Paper N 925. 
Diron, Marie 2008. "Short-term forecasts of euro area real GDP growth: an assessment of realtime performance based on vintage data". Journal of Forecasting. Vol. 27, Issue 5, p.p. 371-390.

Foroni, Claudia and Massimiliano Marcellino. 2013. "A survey of econometric methods for mixed-frequency data. European University Institute. Working paper, 2013/02.

Giacomini, Raffaella and Halbert, White. 2003. "Test of conditional predictive ability". Boston College Working Papers in Economics.

Giannone, Domenico, Lucrezia Reichlin and David Small. 2008. "Nowcasting: The real-time informational content of macroeconomic data". Journal of Monetary Economics 55 (2008), p.p. 665-676.

Higgins, Patrick. 2014. “GDPNow: A Model for GDP Nowcasting” Working Paper 2014-7, Federal Reserve Bank of Atlanta.

Jansen, W. Jos, Xiaowen Jin and Jasper M. de Winter. 2016. "Forecasting and nowcasting real GDP: Comparing statistical models and subjective forecast". International Journal of Forecasting 32 (2016), p.p. 411-436.

Kitchen, John and Ralph Monaco. 2003. "Real-Time Forecasting in Practice". Business Economics, 38 (4): 10-19.

Krief, Tomer. 2011. "A Nowcasting Model for GDP and its Components". Discussion paper $\mathrm{N}^{\circ}$ 2011.01 Research Department, Bank of Israel.

Lago Alves, Sergio Afonso y Angelo Marsiglia Fasolo. 2015. "Not Just Another Mixed Frequency Paper". Working Papers Series, 400. Banco Central do Brasil.

Lahiri, Kajal and George Monokroussos. 2011. "Nowcasting US GDP: The role of ISM Business Surveys". Discussion Papers, University at Albany, SUNY, Department of Economics.

Matheson, Troy. 2011. "New Indicators for Tracking Growth in Real Time". IMF Working Paper $11 / 43$.

Pincheira, Pablo. 2013. "Conditional predictive ability of exchange rate in long run regressions". Revista de Análisis Económico, Vol. 28, N², p.p.3-35.

Shumacher, Christian and Jörg Breitung. 2008. Real-time forecasting of German GDP based on a large factor model with monthly and quarterly data. International Journal of Forecasting, 7 (3), p.p. 375-384.

Stock, J. H., and M. W. Watson. 2002. "Macroeconomic Forecasting Using Diffusion Indexes". Journal of Business and Economic Statistics. Vol 20, N² 2, p.p.147-162.

Tarsidin, Idham and Robbi Nur Rakhman. 2017. "Nowcasting Household Consumption and Investment". Proceedings 2nd ISI Regional Statistics Conference, Indonesia. 


\section{Anexos}

\subsection{Anexo I. Series Estudiadas}

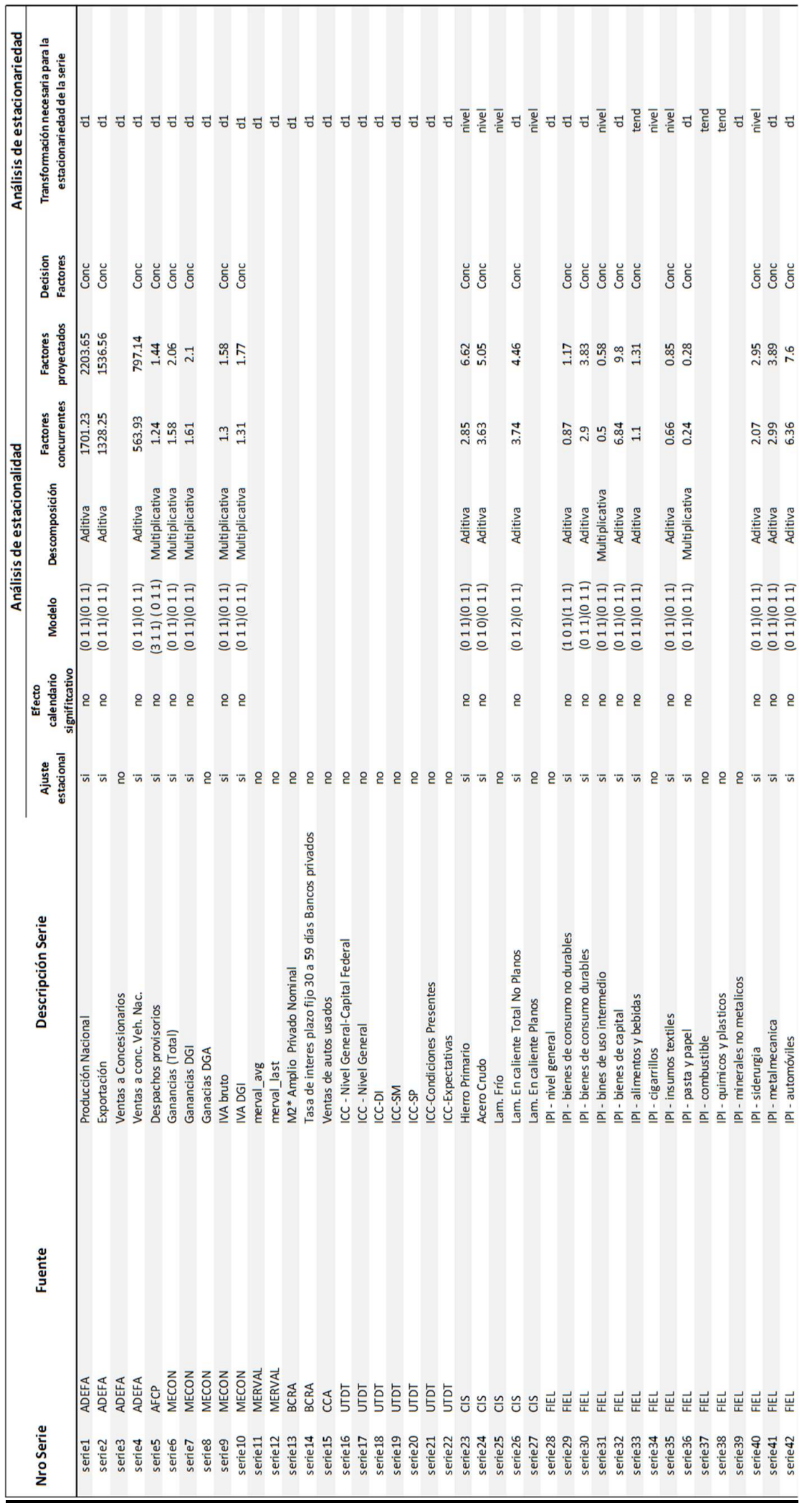




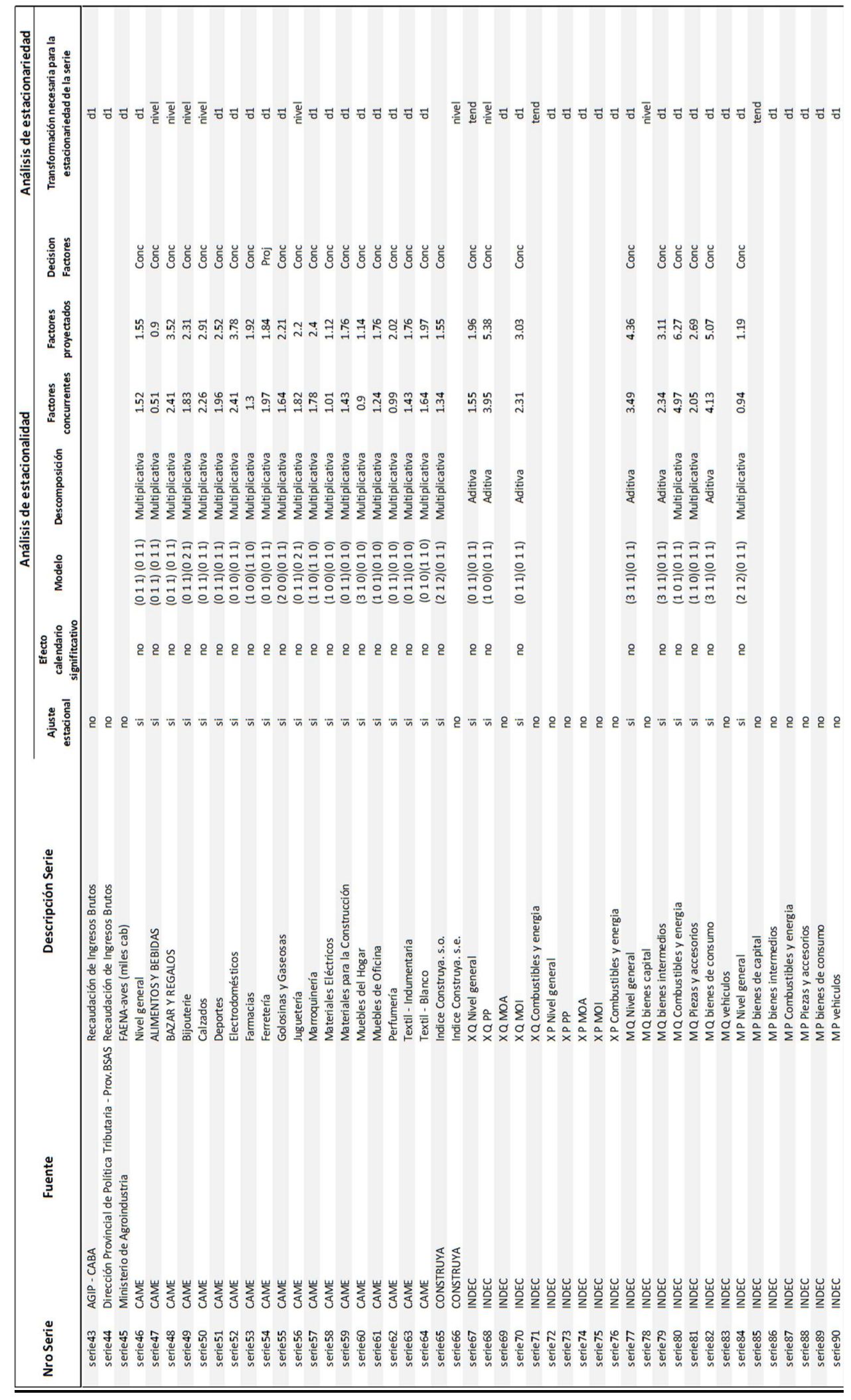




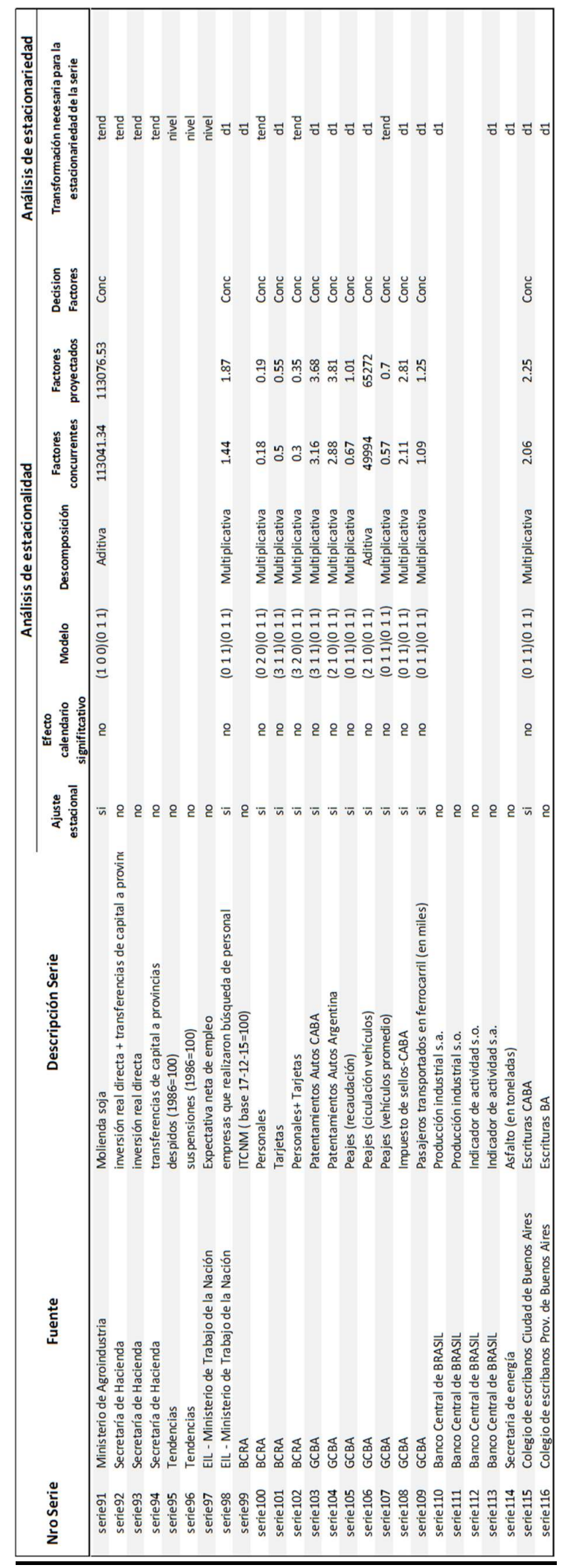




\subsection{Anexo II. Coeficiente de Correlación}

\begin{tabular}{|c|c|c|c|}
\hline Serie & Fuente & $\begin{array}{c}\text { Coeficiente } \\
\text { de Correlación }\end{array}$ & t-Statistic \\
\hline Producción Nacional & ADEFA & 0.43 & 2.96 \\
\hline Ventas a conc. Veh. Nac. & ADEFA & 0.34 & 2.24 \\
\hline Producción industrial s.a. & Banco Central de BRASIL & 0.39 & 2.62 \\
\hline Indicador de actividad s.a. & Banco Central de BRASIL & 0.32 & 2.09 \\
\hline M2* Amplio Privado Nominal & BCRA & 0.39 & 2.59 \\
\hline Tarjetas & BCRA & 0.38 & 2.52 \\
\hline Ferretería & CAME & 0.49 & 3.50 \\
\hline Muebles de Oficina & CAME & 0.49 & 3.46 \\
\hline Electrodomésticos & CAME & 0.48 & 3.38 \\
\hline Materiales para la Construcción & CAME & 0.31 & 2.03 \\
\hline Materiales Eléctricos & CAME & 0.31 & 2.01 \\
\hline Lam. En caliente Total No Planos & CIS & 0.38 & 2.57 \\
\hline Lam. Frío & CIS & 0.33 & 2.15 \\
\hline Lam. En caliente Planos & CIS & 0.32 & 2.11 \\
\hline Escrituras CABA & Colegio de escribanos Ciudad de Buenos Aires & 0.36 & 2.40 \\
\hline Expectativa neta de empleo & EIL - Ministerio de Trabajo de la Nación & 0.38 & 2.56 \\
\hline Empresas que realizaron búsqueda de personal & EIL - Ministerio de Trabajo de la Nación & 0.31 & 2.03 \\
\hline IPI - automóviles & FIEL & 0.49 & 3.43 \\
\hline IPI - bienes de capital & FIEL & 0.40 & 2.68 \\
\hline IPI - bienes de consumo durables & FIEL & 0.39 & 2.58 \\
\hline M Q Nivel general & INDEC & 0.72 & 6.38 \\
\hline M Q bienes intermedios & INDEC & 0.69 & 5.83 \\
\hline M Q Piezas y accesorios & INDEC & 0.64 & 5.20 \\
\hline M Q bienes de consumo & INDEC & 0.55 & 4.08 \\
\hline X P PP & INDEC & 0.47 & 3.26 \\
\hline X P MOI & INDEC & 0.43 & 2.98 \\
\hline X P Nivel general & INDEC & 0.39 & 2.61 \\
\hline$M Q$ vehiculos & INDEC & 0.36 & 2.39 \\
\hline X Q MOI & INDEC & 0.31 & 2.01 \\
\hline Ganancias (Total) & MECON & 0.31 & 2.01 \\
\hline ICC-Condiciones Presentes & UTDT & 0.51 & 3.63 \\
\hline ICC - Nivel General & UTDT & 0.49 & 3.45 \\
\hline ICC-SP & UTDT & 0.48 & 3.37 \\
\hline ICC - Nivel General-Capital Federal & UTDT & 0.43 & 2.96 \\
\hline ICC-Expectativas & UTDT & 0.31 & 1.98 \\
\hline ICC-DI & UTDT & 0.30 & 1.93 \\
\hline
\end{tabular}




\subsection{Anexo III. Componentes Principales}

\section{Factores Grupo 1- Coeficiente: 0.30}

Principal Components Analysis

\begin{tabular}{|c|c|c|c|c|c|}
\hline \multicolumn{6}{|c|}{ Eigenvalues: $($ Sum $=36$, Average $=1)$} \\
\hline Number & Value & Difference & Proportion & $\begin{array}{c}\text { Cumulative } \\
\text { Value }\end{array}$ & $\begin{array}{c}\text { Cumulative } \\
\text { Proportion }\end{array}$ \\
\hline 1 & 12.58344 & 7.038933 & 0.3495 & 12.58344 & 0.3495 \\
\hline 2 & 5.544504 & 1.981026 & 0.1540 & 18.12794 & 0.5036 \\
\hline 3 & 3.563478 & 1.502268 & 0.0990 & 21.69142 & 0.6025 \\
\hline 4 & 2.061211 & 0.142050 & 0.0573 & 23.75263 & 0.6598 \\
\hline 5 & 1.919161 & 0.327167 & 0.0533 & 25.67179 & 0.7131 \\
\hline 6 & 1.591993 & 0.396697 & 0.0442 & 27.26378 & 0.7573 \\
\hline 7 & 1.195296 & 0.112127 & 0.0332 & 28.45908 & 0.7905 \\
\hline 8 & 1.083169 & 0.165967 & 0.0301 & 29.54225 & 0.8206 \\
\hline 9 & 0.917202 & 0.175073 & 0.0255 & 30.45945 & 0.8461 \\
\hline 10 & 0.742129 & 0.044261 & 0.0206 & 31.20158 & 0.8667 \\
\hline 11 & 0.697868 & 0.070236 & 0.0194 & 31.89945 & 0.8861 \\
\hline 12 & 0.627632 & 0.081641 & 0.0174 & 32.52708 & 0.9035 \\
\hline 13 & 0.545991 & 0.089553 & 0.0152 & 33.07307 & 0.9187 \\
\hline 14 & 0.456438 & 0.035586 & 0.0127 & 33.52951 & 0.9314 \\
\hline 15 & 0.420852 & 0.076300 & 0.0117 & 33.95036 & 0.9431 \\
\hline 16 & 0.344552 & 0.097701 & 0.0096 & 34.29492 & 0.9526 \\
\hline 17 & 0.246851 & 0.004752 & 0.0069 & 34.54177 & 0.9595 \\
\hline 18 & 0.242100 & 0.028372 & 0.0067 & 34.78387 & 0.9662 \\
\hline 19 & 0.213727 & 0.015482 & 0.0059 & 34.99759 & 0.9722 \\
\hline 20 & 0.198245 & 0.026659 & 0.0055 & 35.19584 & 0.9777 \\
\hline 21 & 0.171586 & 0.040810 & 0.0048 & 35.36743 & 0.9824 \\
\hline 22 & 0.130776 & 0.031386 & 0.0036 & 35.49820 & 0.9861 \\
\hline 23 & 0.099390 & 0.000309 & 0.0028 & 35.59759 & 0.9888 \\
\hline 24 & 0.099081 & 0.023662 & 0.0028 & 35.69667 & 0.9916 \\
\hline 25 & 0.075419 & 0.012910 & 0.0021 & 35.77209 & 0.9937 \\
\hline 26 & 0.062509 & 0.007800 & 0.0017 & 35.83460 & 0.9954 \\
\hline 27 & 0.054708 & 0.018713 & 0.0015 & 35.88931 & 0.9969 \\
\hline 28 & 0.035995 & 0.012460 & 0.0010 & 35.92530 & 0.9979 \\
\hline 29 & 0.023535 & 0.001565 & 0.0007 & 35.94884 & 0.9986 \\
\hline 30 & 0.021970 & 0.010253 & 0.0006 & 35.97081 & 0.9992 \\
\hline 31 & 0.011717 & 0.002761 & 0.0003 & 35.98253 & 0.9995 \\
\hline 32 & 0.008956 & 0.003461 & 0.0002 & 35.99148 & 0.9998 \\
\hline 33 & 0.005495 & 0.003825 & 0.0002 & 35.99698 & 0.9999 \\
\hline 34 & 0.001670 & 0.000573 & 0.0000 & 35.99865 & 1.0000 \\
\hline 35 & 0.001097 & 0.000841 & 0.0000 & 35.99974 & 1.0000 \\
\hline 36 & 0.000256 & --- & 0.0000 & 36.00000 & 1.0000 \\
\hline
\end{tabular}


Factores Grupo 2- Coeficiente: 0.35

Principal Components Analysis

\begin{tabular}{|c|c|c|c|c|c|}
\hline \multicolumn{6}{|c|}{ Eigenvalues: $($ Sum $=26$, Average $=1)$} \\
\hline Number & Value & Difference & Proportion & $\begin{array}{c}\text { Cumulative } \\
\text { Value }\end{array}$ & $\begin{array}{c}\text { Cumulative } \\
\text { Proportion }\end{array}$ \\
\hline 1 & 9.761444 & 5.855928 & 0.3754 & 9.761444 & 0.3754 \\
\hline 2 & 3.905517 & 1.579478 & 0.1502 & 13.66696 & 0.5257 \\
\hline 3 & 2.326038 & 0.433411 & 0.0895 & 15.99300 & 0.6151 \\
\hline 4 & 1.892627 & 0.385041 & 0.0728 & 17.88563 & 0.6879 \\
\hline 5 & 1.507586 & 0.247845 & 0.0580 & 19.39321 & 0.7459 \\
\hline 6 & 1.259741 & 0.420292 & 0.0485 & 20.65295 & 0.7943 \\
\hline 7 & 0.839449 & 0.137882 & 0.0323 & 21.49240 & 0.8266 \\
\hline 8 & 0.701567 & 0.118276 & 0.0270 & 22.19397 & 0.8536 \\
\hline 9 & 0.583292 & 0.066366 & 0.0224 & 22.77726 & 0.8760 \\
\hline 10 & 0.516926 & 0.081708 & 0.0199 & 23.29419 & 0.8959 \\
\hline 11 & 0.435218 & 0.010352 & 0.0167 & 23.72941 & 0.9127 \\
\hline 12 & 0.424866 & 0.043545 & 0.0163 & 24.15427 & 0.9290 \\
\hline 13 & 0.381321 & 0.092261 & 0.0147 & 24.53559 & 0.9437 \\
\hline 14 & 0.289061 & 0.023469 & 0.0111 & 24.82466 & 0.9548 \\
\hline 15 & 0.265592 & 0.061268 & 0.0102 & 25.09025 & 0.9650 \\
\hline 16 & 0.204325 & 0.021848 & 0.0079 & 25.29457 & 0.9729 \\
\hline 17 & 0.182476 & 0.036019 & 0.0070 & 25.47705 & 0.9799 \\
\hline 18 & 0.146457 & 0.003560 & 0.0056 & 25.62351 & 0.9855 \\
\hline 19 & 0.142897 & 0.050381 & 0.0055 & 25.76640 & 0.9910 \\
\hline 20 & 0.092516 & 0.039249 & 0.0036 & 25.85892 & 0.9946 \\
\hline 21 & 0.053267 & 0.013523 & 0.0020 & 25.91219 & 0.9966 \\
\hline 22 & 0.039744 & 0.011645 & 0.0015 & 25.95193 & 0.9982 \\
\hline 23 & 0.028099 & 0.016493 & 0.0011 & 25.98003 & 0.9992 \\
\hline 24 & 0.011605 & 0.005245 & 0.0004 & 25.99163 & 0.9997 \\
\hline 25 & 0.006361 & 0.004356 & 0.0002 & 25.99800 & 0.9999 \\
\hline 26 & 0.002005 & --- & 0.0001 & 26.00000 & 1.0000 \\
\hline
\end{tabular}


Factores Grupo 3- Coeficiente: 0.40

Principal Components Analysis

\begin{tabular}{|c|c|c|c|c|c|}
\hline \multicolumn{6}{|c|}{ Eigenvalues: $($ Sum $=19$, Average $=1)$} \\
\hline Number & Value & Difference & Proportion & $\begin{array}{c}\text { Cumulative } \\
\text { Value }\end{array}$ & $\begin{array}{l}\text { Cumulative } \\
\text { Proportion }\end{array}$ \\
\hline 1 & 7.807496 & 4.051372 & 0.4109 & 7.807496 & 0.4109 \\
\hline 2 & 3.756123 & 1.983448 & 0.1977 & 11.56362 & 0.6086 \\
\hline 3 & 1.772676 & 0.457612 & 0.0933 & 13.33629 & 0.7019 \\
\hline 4 & 1.315064 & 0.265941 & 0.0692 & 14.65136 & 0.7711 \\
\hline 5 & 1.049123 & 0.375372 & 0.0552 & 15.70048 & 0.8263 \\
\hline 6 & 0.673751 & 0.130484 & 0.0355 & 16.37423 & 0.8618 \\
\hline 7 & 0.543267 & 0.116622 & 0.0286 & 16.91750 & 0.8904 \\
\hline 8 & 0.426645 & 0.045551 & 0.0225 & 17.34414 & 0.9128 \\
\hline 9 & 0.381094 & 0.034525 & 0.0201 & 17.72524 & 0.9329 \\
\hline 10 & 0.346568 & 0.084160 & 0.0182 & 18.07181 & 0.9511 \\
\hline 11 & 0.262408 & 0.016564 & 0.0138 & 18.33421 & 0.9650 \\
\hline 12 & 0.245844 & 0.075366 & 0.0129 & 18.58006 & 0.9779 \\
\hline 13 & 0.170478 & 0.054172 & 0.0090 & 18.75054 & 0.9869 \\
\hline 14 & 0.116306 & 0.051215 & 0.0061 & 18.86684 & 0.9930 \\
\hline 15 & 0.065091 & 0.033324 & 0.0034 & 18.93193 & 0.9964 \\
\hline 16 & 0.031767 & 0.009933 & 0.0017 & 18.96370 & 0.9981 \\
\hline 17 & 0.021834 & 0.009876 & 0.0011 & 18.98553 & 0.9992 \\
\hline 18 & 0.011958 & 0.009450 & 0.0006 & 18.99749 & 0.9999 \\
\hline 19 & 0.002508 & --- & 0.0001 & 19.00000 & 1.0000 \\
\hline
\end{tabular}




\subsection{Anexo IV. Estimaciones}

\section{Nowcast G1}

Dependent Variable: INVERSION

\begin{tabular}{lrcrr}
\hline \hline \multicolumn{1}{c}{ Variable } & Coefficient & Std. Error & t-Statistic & Prob. \\
\hline \hline C & 0.005149 & 0.003855 & 1.335846 & 0.1910 \\
INVERSION(-1) & 0.138296 & 0.074182 & 1.864291 & 0.0715 \\
$\quad$ PC1_1 & 0.012301 & 0.001274 & 9.657935 & 0.0000 \\
DMAR08 & 0.065086 & 0.006029 & 10.79553 & 0.0000 \\
\multicolumn{1}{c}{ DJUN11 } & -0.103707 & 0.007930 & -13.07820 & 0.0000 \\
\hline \hline R-squared & 0.783084 & Mean dependent var & 0.006800 \\
Adjusted R-squared & 0.755970 & S.D. dependent var & & 0.058749 \\
S.E. of regression & 0.029022 & Akaike info criterion & -4.116471 \\
Sum squared resid & 0.026952 & Schwarz criterion & -3.898780 \\
Log likelihood & 81.15472 & Hannan-Quinn criter. & -4.039725 \\
F-statistic & 28.88071 & Durbin-Watson stat & 2.007653 \\
Prob(F-statistic) & 0.000000 & & \\
\hline \hline
\end{tabular}

\section{Nowcast G2}

Dependent Variable: INVERSION

\begin{tabular}{lrlrr}
\hline \hline \multicolumn{1}{c}{ Variable } & Coefficient & Std. Error & t-Statistic & Prob. \\
\hline \hline \multicolumn{1}{c}{ C } & 0.006614 & 0.003488 & 1.896055 & 0.0665 \\
\multicolumn{1}{c}{ INVERSION(-1) } & 0.153518 & 0.076255 & 2.013226 & 0.0521 \\
$\quad$ PC1_2 & 0.013373 & 0.001291 & 10.35517 & 0.0000 \\
DJUN11 & -0.099372 & 0.006938 & -14.32308 & 0.0000 \\
\multicolumn{1}{c}{ DMAR08 } & 0.054529 & 0.005550 & 9.825579 & 0.0000 \\
\hline \hline R-squared & 0.779750 & Mean dependent var & 0.007716 \\
Adjusted R-squared & 0.753838 & S.D. dependent var & 0.057321 \\
S.E. of regression & 0.028440 & Akaike info criterion & -4.162854 \\
Sum squared resid & 0.027500 & Schwarz criterion & -3.949577 \\
Log likelihood & 86.17565 & Hannan-Quinn criter. & -4.086332 \\
F-statistic & 30.09244 & Durbin-Watson stat & 2.089489 \\
Prob(F-statistic) & 0.000000 & & \\
\hline \hline
\end{tabular}




\section{$\underline{\text { Nowcast G3 }}$}

Dependent Variable: INVERSION

\begin{tabular}{lrcrr}
\hline \hline \multicolumn{1}{c}{ Variable } & Coefficient & Std. Error & t-Statistic & Prob. \\
\hline \hline C & 0.012101 & 0.004252 & 2.846172 & 0.0076 \\
INVERSION(-1) & 0.123251 & 0.080626 & 1.528668 & 0.1359 \\
PC1_3 & 0.014276 & 0.001548 & 9.223689 & 0.0000 \\
DMAR09 & -0.080891 & 0.027689 & -2.921415 & 0.0062 \\
DJUN11 & -0.100521 & 0.025751 & -3.903537 & 0.0004 \\
\multicolumn{1}{c}{ DSEPT14 } & -0.065617 & 0.025298 & -2.593824 & 0.0140 \\
\hline \hline R-squared & 0.835708 & Mean dependent var & 0.007716 \\
Adjusted R-squared & 0.810815 & S.D. dependent var & 0.057321 \\
S.E. of regression & 0.024932 & Akaike info criterion & -4.404689 \\
Sum squared resid & 0.020513 & Schwarz criterion & -4.148757 \\
Log likelihood & 91.89144 & Hannan-Quinn criter. & -4.312863 \\
F-statistic & 33.57228 & Durbin-Watson stat & 2.085440 \\
Prob(F-statistic) & 0.000000 & & \\
\hline \hline
\end{tabular}

$\underline{\mathbf{A R}}$

Dependent Variable: INVERSION

\begin{tabular}{lrlrr}
\hline \hline \multicolumn{1}{c}{ Variable } & Coefficient & Std. Error & t-Statistic & Prob. \\
\hline \multicolumn{1}{c}{ C } & 0.011753 & 0.007656 & 1.535261 & 0.1337 \\
\multicolumn{1}{c}{ INVERSION(-1) } & 0.275602 & 0.122414 & 2.251385 & 0.0307 \\
$\quad$ DDIC08 & -0.119750 & 0.007728 & -15.49642 & 0.0000 \\
\multicolumn{1}{c}{ DMAR09 } & -0.143933 & 0.014898 & -9.661175 & 0.0000 \\
\hline \hline R-squared & 0.437406 & Mean dependent var & 0.007716 \\
Adjusted R-squared & 0.389184 & S.D. dependent var & 0.057321 \\
S.E. of regression & 0.044799 & Akaike info criterion & -3.276343 \\
Sum squared resid & 0.070244 & Schwarz criterion & -3.105721 \\
Log likelihood & 67.88869 & Hannan-Quinn criter. & -3.215125 \\
F-statistic & 9.070612 & Durbin-Watson stat & 1.830677 \\
Prob(F-statistic) & 0.000140 & & \\
\hline \hline
\end{tabular}

